DESY-11-065

\title{
Effective action for the Regge processes in gravity
}

\author{
L.N. Lipatov \\ St. Petersburg State University, \\ Petersburg Nuclear Physics Institute, \\ Gatchina, 188300, St.Petersburg, Russia, \\ Universität Hamburg, \\ II. Institut für Theoretische Physik, \\ Luruper Chaussee,149, D-22761 Hamburg
}

\begin{abstract}
It is shown, that the effective action for the reggeized graviton interactions can be formulated in terms of the reggeon fields $A^{++}$and $A^{--}$and the metric tensor $g_{\mu \nu}$ in such a way, that it is local in the rapidity space and has the property of general covariance. The corresponding effective currents $j^{-}$and $j^{+}$satisfy the Hamilton-Jacobi equation for a massless particle moving in the gravitational field. These currents are calculated explicitly for the shock wave-like fields and a variation principle for them is formulated. As an application, we reproduce the effective lagrangian for the multi-regge processes in gravity together with the graviton Regge trajectory in the leading logarithmic approximation with taking into account supersymmetric contributions.
\end{abstract}




\section{Introduction}

In the Regge pole model the scattering amplitude at large energies $\sqrt{s}$ and fixed momentum transfers $\sqrt{-t}$ has the form $[1]$

$$
A_{\text {Regge }}^{p}(s, t)=\xi_{p}(t) s^{1+\omega_{p}(t)} \gamma^{2}(t), \xi_{p}(t)=e^{-i \pi \omega_{p}(t)}-p,
$$

where $p= \pm 1$ is the signature of the reggeon with the trajectory $\omega_{p}(t)$. The Pomeron is the Regge pole of the $t$-channel partial waves $f_{\omega}(t)$ with vacuum quantum numbers and the positive signature describing an approximately constant behaviour of total cross-sections for the hadron-hadron scattering. S. Mandelstam demonstrated, that the Regge poles generate cut singularities in the $\omega$-plane [2]. These singularities appear as a result of the analytic continuation of the multi-particle unitarity condition [3]. They correspond to scattering states of the reggeons. Using the locality of the reggeon interactions in the rapidity space, V. Gribov constructed an effective $(2+1)$ Pomeron field model [4].

On the other hand, it was discovered, that in some field theories the elementary particles become reggeons after taking into account radiative corrections. The simplest example of this phenomenon is the electron reggeization in quantum electrodynamics with a massive photon [5]. Using the counting rules suggested in Ref. [6] the vector boson reggeization in the gauge models with the Higgs mechanism was also established [7].

In the leading logarithmic approximation (LLA) the scattering amplitude at high energies in QCD has the Regge form [8]

$$
M_{A B}^{A^{\prime} B^{\prime}}(s, t)=\left.M_{A B}^{A^{\prime} B^{\prime}}(s, t)\right|_{B o r n} s^{\omega(t)},
$$

where $M_{\text {Born }}$ is the Born amplitude and the gluon Regge trajectory is given below

$$
\omega\left(-|q|^{2}\right)=-\frac{\alpha_{s} N_{c}}{4 \pi^{2}} \int d^{2} k \frac{|q|^{2}}{|k|^{2}|q-k|^{2}} \approx-\frac{\alpha_{s} N_{c}}{2 \pi} \ln \frac{\left|q^{2}\right|}{\lambda^{2}}
$$

where $\lambda$ is a gluon mass, introduced for the infrared regularization.

In the multi-Regge kinematics, where the pair energies $\sqrt{s_{k}}$ of the produced gluons are large in comparison with momentum transfers $\left|q_{i}\right|$ the production amplitudes in LLA are constructed from products of the Regge factors $s_{k}^{\omega\left(t_{k}\right)}$ and effective reggeon-reggeon-gluon vertices $C_{\mu}\left(q_{r}, q_{r+1}\right)$ [8]. The amplitudes satisfy the Steinmann relations and the $s$-channel unitarity leading to bootstrap equations [8].

The knowledge of $M_{2 \rightarrow 2+n}$ allows us to construct the BFKL equation for the Pomeron wave function [8]

$$
E \Psi\left(\vec{\rho}_{1}, \vec{\rho}_{2}\right)=H_{12} \Psi\left(\vec{\rho}_{1}, \vec{\rho}_{2}\right), \sigma_{t} \sim s^{\Delta}, \Delta=-\frac{\alpha_{s} N_{c}}{2 \pi} E_{0}
$$

Here $H_{12}$ is the BFKL Hamiltonian and $\Delta$ is the Pomeron intercept. The operator $H_{12}$ has the property of the holomorphic separability [9] and is invariant under the Möbius transformations [10]

$$
\rho_{k} \rightarrow \frac{a \rho_{k}+b}{c \rho_{k}+d}
$$


The generalization of eq. (4) to a composite state of several gluons [11] in the multi-color QCD leads to an integrable XXX model [12] having a duality symmetry [13].

The next-to-leading correction to the BFKL kernel in QCD is also calculated [14]. Its eigenvalue contains non-analitic terms proportional to $\delta_{n, 0}$ and $\delta_{n, 2}$, where $n$ is the conformal spin of the Möbius group. But in the case of the $N=4$ extended supersymmetric gauge model these Kronecker symbols are canceled leading to an expression having the properties of the hermitian separability [15] and maximal transcendentality [16]. The last property allowed to calculate the anomalous dimensions of twist-two operators up to three loops [17]. It turns out, that evolution equations for the so-called quasi-partonic operators are integrable in $N=4$ SUSY at the multi-color limit [18]. The $N=4$ four-dimensional conformal field theory due to the Maldacena guess is equivalent to the superstring living on the anti-deSitter 10-dimensional space $[19,20,21]$. Therefore the Pomeron in N=4 SUSY is equivalent to the reggeized graviton in this space. This equivalence gives a possibility to calculate the intercept of the BFKL Pomeron at large coupling constants [17, 22]

$$
j=2-\Delta, \Delta=\frac{1}{2 \pi} \hat{a}^{-1 / 2}, a=\frac{g^{2} N_{c}}{16 \pi^{2}} .
$$

The duality between the BFKL Pomeron and the reggeized graviton means, that the Pomeron calculus could be described in a framework of the approach based on the effective action for the reggeized gravitons. It is one of the reasons, why we investigate in this paper the gravity at high energies.

To begin with, let us remind the effective field theory for reggeized gluons [23]. The corresponding effective action is local in the rapidity space

$$
y=\frac{1}{2} \ln \frac{\epsilon_{k}+|k|}{\epsilon_{k}-|k|},\left|y-y_{0}\right|<\eta, \eta<<\ln s .
$$

We introduce the anti-hermitian fields $v_{\mu}$ for the usual gluons and the gauge invariant fields $A_{ \pm}$describing the production and annihilation of the reggeized gluons

$$
v_{\mu}(x)=-i T^{a} v_{\mu}^{a}(x), A_{ \pm}(x)=-i T^{a} A_{ \pm}^{a}(x), \delta A_{ \pm}(x)=0,
$$

where $T^{a}$ are generators of the gauge group in the adjoint representation. The fields $A_{ \pm}$ satisfy the kinematical constraints

$$
\partial_{-} A_{+}=\partial_{+} A_{-}=0
$$

The effective action for a cluster of particles with approximately equal rapidities has the form

$$
S=\int d^{4} x\left(L_{Q C D}+\operatorname{Tr}\left(V_{+} \partial_{\mu}^{2} A_{-}+V_{-} \partial_{\mu}^{2} A_{+}\right)\right)
$$

where $L_{Q C D}$ is the usual QCD action and the effective currents $V_{ \pm}$are given below

$$
V_{+}=-\frac{1}{g} \partial_{+} P \exp \left(-\frac{g}{2} \int_{-\infty}^{x^{+}} v_{+}\left(x^{\prime}\right) d\left(x^{\prime}\right)^{+}\right)=v_{+}-g v_{+} \frac{1}{\partial_{+}} v_{+}+\ldots
$$

The Feynman rules for this action are derived in ref. [24]. The effective action approach gives a possibility to construct various reggeon vertices needed to calculate NLO and NNLO corrections to the BFKL kernel. 
Another application of the effective action for the gauge models is a verification of the BDS ansatz [25] for the inelastic amplitudes in $N=4$ SUSY. It was shown [26, 27], that the BDS amplitude $M_{2 \rightarrow 4}^{B D S}$ should be multiplied by the factor containing the contribution of the Mandelstam cut in LLA. In the two-loop approximation this factor can be found also from properties of analyticity and factorization [28] or directly from recently obtained exact result for $M_{2 \rightarrow 4}$ [29]. In a general case the wave function for the Mandelstam singularity satisfies the Schrödinger equation for an open integrable Heisenberg spin chain [30].

Below we apply the approach based on the effective action for reggeons to the case of the high energy gravity. The graviton reggeization was established initially with the use of the t-channel unitarity [31]. Later it was shown [32], that in LLA the graviton Regge trajectory is finite in the ultraviolet region only in the $N=4$ supergravity. At other gravity models the corresponding $t$-channel partial wave $f_{j}(t)$ has a Regge cut singularity corresponding to the double-logarithmic asymptotics [32]. Also some effective vertices for reggeized graviton interactions were calculated [33]. These results were verified by the authors of Ref. [34] in their study of the gravity at Planckian energies. An effective field theory for the $S$-matrix in gravity with the multi-Regge unitarity was constructed in Ref. [35], which allowed to investigate the gravitational collapse at the high energy particle scattering [36]. The new results on the high energy scattering in gravity and supergravity and related references can be found in the paper [37].

\section{Reggeon fields in gravity}

It is natural to construct the theory of high energy processes in gravity in terms of the reggeized gravitons and their interactions, because in this case the scattering amplitudes will satisfy automatically the $t$-channel unitarity. The $S$-channel unitarity will be incorporated in the reggeon vertices. In particular, the so-called bootstrap relations in QCD are consequences of the multi-particle $S$-channel unitarity. We begin with the introduction of the fields describing the usual and reggeized gravitons.

The Hilbert-Einstein action for gravity has the form [38]

$$
S=S_{\text {grav }}+S_{m}
$$

where $S_{\text {grav }}$ and $S_{m}$ describe the interaction of the gravity field $g_{\mu \nu}(x)$ and the matter fields, respectively. Both contributions are invariant under the general coordinate transformation. For the metric tensor $g_{\mu \nu}$, entering in the invariant interval

$$
(d s)^{2}=\sum_{\mu \nu} g_{\mu \nu} d x^{\mu} d x^{\nu}
$$

this transformation in the infinitesimal form is given below

$$
\delta g_{\mu \nu}(x)=D_{\mu} \chi_{\nu}(x)+D_{\nu} \chi_{\mu}(x)
$$

where $\chi_{\sigma}(x)$ is a small local parameter and $D_{\sigma}$ is the covariant derivative defined below

$$
D_{\mu} \chi_{\nu}(x)=\partial_{\mu} \chi_{\nu}(x)-\Gamma_{\mu \nu}^{\rho} \chi_{\rho}(x) .
$$


Here $\partial_{\mu}$ is the partial derivative in $x^{\mu}$ and $\Gamma_{\mu \nu}^{\rho}$ is the Christoffel symbol

$$
\Gamma_{\mu \nu}^{\rho}=\frac{1}{2} g^{\rho \sigma}\left(\partial_{\mu} g_{\sigma \nu}+\partial_{\nu} g_{\mu \sigma}-\partial_{\sigma} g_{\mu \nu}\right) .
$$

Note, that in the Minkowski space the corresponding invariant interval is

$$
(d s)^{2}=\eta_{\mu \nu} d x^{\mu} d x^{\nu},
$$

where the Lorentz tensor $\eta^{\mu \nu}$ has the signature $(+---)$.

The action for the pure gravity can be written as follows

$$
S_{\text {grav }}=-\frac{1}{2 \kappa} \int d^{4} x \sqrt{-g} R,
$$

where

$$
g=\operatorname{Det}\left(g_{\mu \nu}\right)
$$

and the Einstein parameter $\kappa$ is proportional to the Newton constant $\gamma$

$$
\kappa=8 \pi \gamma
$$

The scalar curvature $R$ is related to the Riemann tensor by the contraction of indices

$$
R=R_{\mu \nu} g^{\mu \nu} .
$$

In turn, $R_{\mu \nu}$ is expressed in terms of the Riemann tensor of the fourth rank

$$
R_{\mu \nu}=R_{\mu, \sigma \nu}^{\sigma}, R_{\mu, \alpha \beta}^{\sigma}=\partial_{\beta} \Gamma_{\mu \alpha}^{\sigma}-\partial_{\alpha} \Gamma_{\mu \beta}^{\sigma}+\Gamma_{\mu \alpha}^{\rho} \Gamma_{\rho \beta}^{\sigma}-\Gamma_{\mu \beta}^{\rho} \Gamma_{\rho \alpha}^{\sigma} .
$$

The matter action can be written as an integral from the Lagrangian $L_{m}$

$$
S_{m}=\int d^{4} x \sqrt{-g} L_{m} .
$$

Its variation in the metric tensor is expressed in terms of the energy-momentum tensor $T^{\mu \nu}$

$$
\delta S_{m}=\frac{1}{2} \int d^{4} x \sqrt{-g} \delta g_{\mu \nu} T^{\mu \nu},
$$

which is conserved

$$
D_{\mu} T^{\mu \nu}=0 .
$$

Performing also the variation of the gravity action

$$
\delta S_{\text {grav }}=\frac{1}{2 \kappa} \int d^{4} x \sqrt{-g} \delta g_{\mu \nu} G^{\mu \nu}, G^{\mu \nu} \equiv R^{\mu \nu}-\frac{1}{2} g^{\mu \nu} R,
$$

we obtain from the stationarity condition for the total action $\delta S=0$ the Einstein equations

$$
G^{\mu \nu}=-\kappa T^{\mu \nu}
$$


Due to the general covariance of the action $S_{\text {grav }}$ the left hand side of this equality satisfies the identity

$$
D_{\mu} G^{\mu \nu}=0
$$

which is compatible with the conservation of the energy-momentum tensor $T^{\mu \nu}$.

We want to construct the interaction of the Reggeized gravitons and usual gravitons to describe quasi-multi-Regge processes at high energies. In these processes intermediate particles are produced in clusters with fix invariant masses $m_{r}$. The particles in each cluster have approximately the same rapidities $y \approx y_{r}$ in the interval

$$
\left|y-y_{r}\right|<\eta
$$

where the intermediate parameter $\eta$ is assumed to satisfy the inequalities

$$
\ln s \gg \eta \gg 1 \text {. }
$$

The relative rapidities of different clusters produced in the multi-Regge kinematics are considered to be large

$$
\ln s \gg y_{r}-y_{r-1} \gg \eta \text {. }
$$

The interaction between the clusters is performed through an exchange of the reggeized gravitons described by two additional fields $A^{++}$and $A^{--}$. These new fields correspond to the reggeon emission and absorption in the crossing channel. In our quasi-multi-Regge kinematics corresponding to the strongly ordered rapidities they satisfy the following kinematical constraints (cf. (32))

$$
\partial_{+} A^{++}=0, \partial_{-} A^{--}=0
$$

where

$$
\partial_{ \pm}=n_{ \pm}^{\sigma} \partial_{\sigma}, n_{ \pm}^{2}=0, n_{+} n_{-}=1
$$

and the light-cone vectors $n_{ \pm}$are expressed in terms of momenta $p_{A}, p_{B}$ of colliding particles

$$
n_{+}=p_{A} \sqrt{\frac{2}{s}}, n_{-}=p_{B} \sqrt{\frac{2}{s}} .
$$

The above constraints for $A^{ \pm}$follow from the fact that the Sudakov variables $\alpha_{r}, \beta_{r}$ for the produced cluster momentum

$$
k_{r}=\beta_{r} p_{A}+\alpha_{r} p_{B}+k_{r \perp}, k_{r}^{2}=s \alpha_{r} \beta_{r}-\mathbf{k}_{r \perp}^{2}=m_{r}^{2}
$$

are strongly ordered

$$
1 \gg \beta_{1} \gg \beta_{2} \gg \ldots \gg \beta_{n}, 1 \gg \alpha_{n} \gg \alpha_{n-1} \gg \ldots \gg \beta_{1}, s \alpha_{r} \beta_{r} \sim \mathbf{k}_{r \perp}^{2} \sim m_{r}^{2} .
$$

We derive in next sections the effective action for high energy processes in gravity. This action describes the interaction of gravitons inside each cluster with neighboring reggeons having approximately the same rapidities. In this case apart from the usual Hilbert-Einstein action $S_{\text {grav }}$ one should introduce an additional contribution $\Delta S$ containing the linear combination of the reggeon fields $A^{++}$and $A^{--}$considered them as external sources

$$
S_{e f f}=S_{g r a v}+\Delta S
$$


It is well known, that the reggeon describes a family of particles with different spins and masses lying on the Regge trajectory. The reggeized graviton can be viewed as a natural generalization of the usual massless graviton with the spin $j=2$. Therefore it is natural to consider the functions $A^{++}$and $A^{--}$as fields invariant under general covariant transformations

$$
\delta A^{++}=\delta A^{--}=0
$$

with the corresponding local parameters $\chi$ decreasing at large $x$. Of course, $A^{++}$and $A^{--}$ are transformed under the global Poincare group. The induced contribution $\Delta S$ can be written in the form

$$
\Delta S=-\frac{1}{2 \kappa} \int d^{4} x\left(j_{++} \frac{\partial_{\mu}^{2} A^{++}}{2}+j_{--} \frac{\partial_{\mu}^{2} A^{--}}{2}\right) .
$$

Here the Laplacian operators $\partial_{\mu}^{2}$ are introduced to avoid simultaneous singularities in the overlapping direct and crossing channels because they cancel neighboring reggeon propagators $1 / q^{2}$. These propagators appear due to a kinetic term bilinear in the fields $A^{ \pm \pm}$. As it is shown below, due to general covariance the currents $j_{++}$and $j_{--}$contain the non-local operators $\partial_{+}^{-1}$ and $\partial_{-}^{-1}$ which should be interpreted as propagators of the particles in other clusters emitting the gravitons into the given rapidity interval $\eta$.

In the perturbation theory the currents $j_{++}, j_{--}$contain contributions linear in the metric tensor $g_{\mu \nu}$

$$
j_{++} \approx g_{++}+\ldots, j_{--} \approx g_{--}+\ldots .
$$

The corresponding solution of the Einstein equation for $g^{\mu \nu}$ in the external fields $A^{++}$and $A^{--}$will have the form

$$
g^{\mu \nu}=\eta^{\mu \nu}+\delta_{+}^{\mu} \delta_{+}^{\nu} A^{++}+\delta_{-}^{\mu} \delta_{-}^{\nu} A^{--}+O\left(A^{2}\right) .
$$

Therefore the reggeon fields $A^{++}$and $A^{--}$can be considered as classical components of the gravity field.

The induced term $\Delta S$ should be invariant under general coordinate transformations providing that $A^{ \pm \pm}$satisfy the kinematical constraints (32). As it was argued above, the currents $j_{++}$and $j_{--}$describe the graviton emission into the given interval of rapidities from other clusters having different rapidities. In an accordance with the condition $\partial_{ \pm} A^{ \pm \pm}=0$ two neighboring reggeons for the cluster $r$ have the momentum components $k_{r}^{ \pm}$which are transferred almost completely to the particles in the cluster. These momenta are shared by the particles in other clusters with higher values of $k^{ \pm}$. Because the currents $j_{ \pm}$are universal, for their calculation one can consider an arbitrary process in the external field having particles with the larger components $k^{ \pm}$of their momenta.

\section{General covariance of the effective action}

To calculate the effective currents $j_{++}$and $j_{--}$we use their invariance under the general coordinate transformations up to the total derivatives in $x^{+}$and $x^{-}$, taking into account the fact that the fields $A^{++}$and $A^{--}$are invariant under these transformations and satisfy additional constraints

$$
\partial_{+} A^{++}=\partial_{-} A^{--}=0
$$


As a gravitational field we chose the tensor $h_{\mu \nu}$ in the following decomposition of the covariant metric tensor

$$
g_{\mu \nu}=\eta_{\mu \nu}+h_{\mu \nu} .
$$

The components of the contravariant metric tensor $g^{\mu \nu}$ can be found from the linear equation

$$
g^{\mu \sigma} g_{\sigma \nu}=\delta_{\nu}^{\mu}
$$

and are obtained by the perturbation expansion

$$
g^{\mu \nu}=\eta^{\mu \nu}-h_{\mu \nu}+h_{\mu \rho} h_{\rho \nu}-h_{\mu \rho} h_{\rho \delta} h_{\delta \nu}+\ldots
$$

Note, that for the tensor $h_{\mu \nu}$ and its derivatives we shall use only lower components implying the Minkowski signature in summation over the repeated indices. The effective currents $j_{++}$ and $j_{--}$can be calculated in the perturbation series over the tensor components $h_{\mu \nu}$. For example, $j_{++}$can be presented as follows

$$
j_{++}=h_{++}+P_{++}^{(2)}(h)+P_{++}^{(3)}(h)+\ldots,
$$

where the polynomials $P_{++}^{(k)}$ can contain derivatives $\partial_{\sigma}$ and integral operators $1 / \partial_{+}$acting on the fields $h$. Furthermore, generally we differ the components $h_{++}, h_{\sigma+}$ and $h_{\rho \sigma}$. The corresponding set of recurrent equations for the homogeneous polynomials $P_{++}^{(n)}$ are obtained from the general covariance of the induced action using the infinitesimal transformations with parameters $\chi_{\rho}$ and $\chi_{+}$

$$
\begin{gathered}
\frac{\delta P_{++}^{(n)}}{\delta h_{\rho \sigma}} 2 \partial_{\sigma} \chi_{\rho}+\frac{\delta P_{++}^{(n)}}{\delta h_{\rho+}} \partial_{+} \chi_{\rho}=\sum_{k=1}^{n-1} \frac{\delta P_{++}^{(k)}}{\delta h_{\rho \sigma}} 2 \Gamma_{\rho \sigma}^{\nu} \chi_{\nu}, \\
\frac{\delta P_{++}^{(n)}}{\delta h_{++}} 2 \partial_{+} \chi_{+}+\frac{\delta P_{++}^{(n)}}{\delta h_{\rho+}} \partial_{\rho} \chi_{+}=0 .
\end{gathered}
$$

In the right hand side of the first equation one should leave only the terms of the order $n$ in the perturbation series over $h_{\rho \sigma}$ using the above expansion for $g^{\mu \nu}$. It is implied also in these equations, that after the differentiation over $h_{\rho \sigma}, h_{\rho+}$ and $h_{++}$the corresponding tensor components in $P_{++}$should be replaced by the subsequent factors.

The second equation can be easily solved. Namely, $P_{++}^{(n)}$ should contain the dependence from $h_{\sigma+}$ and $h_{++}$only in the form of the following linear combination

$$
X_{\sigma+}=X_{+\sigma}=h_{\sigma+}-\frac{1}{2} \frac{\partial_{\sigma}}{\partial_{+}} h_{++} .
$$

Moreover, we can add to the solution of the first equation an arbitrary function of another linear combination

$$
Z_{\rho \sigma}=h_{\rho \sigma}-2 \frac{\partial_{\rho}}{\partial_{+}} h_{\sigma+}
$$

It is convenient to introduce two independent variables: $X_{\sigma+}$ and

$$
Y_{\rho \sigma}=h_{\rho \sigma}-2 \frac{\partial_{\rho}}{\partial_{+}} h_{\sigma+}+\frac{\partial_{\rho} \partial_{\sigma}}{\partial_{+}^{2}} h_{++}=h_{\rho \sigma}-2 \frac{\partial_{\rho}}{\partial_{+}} X_{\sigma+}
$$


Then the left hand sides of the above equations do not contain the derivative in $Y_{\rho \sigma}$ and the first equation can be written as follows

$$
\frac{\partial P_{++}^{(n)}}{\partial X_{\sigma+}} \partial_{+} \chi_{\sigma}=\sum_{k=1}^{n-1} \frac{\delta P_{++}^{(k)}}{\delta h_{\rho \sigma}} 2 \Gamma_{\rho \sigma}^{\nu} \chi_{\nu}
$$

Here the right hand side should be expressed in terms of the variables $X_{\sigma+}$ and $Y_{\rho \sigma}$. In particular using

$$
P_{++}^{(1)}=h_{++}
$$

for $P_{++}^{(2)}$ one can obtain the equation

$$
\frac{\partial P_{++}^{(2)}}{\partial X_{\sigma+}} \partial_{+} \chi_{\sigma}=-2 X_{\sigma+} \partial_{+} \chi_{\sigma}
$$

where the following relation was used

$$
\delta h_{++}=2 \partial_{+} \chi_{+}-2 \chi^{\sigma} \partial_{+} X_{\sigma+}
$$

with the subsequent integration over $x_{+}$by parts. Note, that the first term $2 \partial_{+} \chi_{+}$in $\delta h_{++}$ gives a vanishing contribution to the induced action $\Delta S$ in this order due to the kinematical constraint

$$
\partial_{+} A^{++}=0
$$

Therefore from eq. (53) we obtain

$$
P_{++}^{(2)}=-X_{\sigma+}^{2}
$$

To find $P_{++}$in upper orders of the perturbation theory one should use the following relations

$$
\begin{gathered}
\delta h_{\sigma+}=\partial_{\sigma} \chi_{+}+\partial_{+} \chi_{\sigma}-\chi^{\rho}\left(\partial_{+} h_{\sigma \rho}+\partial_{\sigma} h_{+\rho}-\partial_{\rho} h_{+\sigma}\right) \\
\delta h_{\sigma \nu}=\partial_{\sigma} \chi_{\nu}+\partial_{\nu} \chi_{\sigma}-\chi^{\rho}\left(\partial_{\nu} h_{\sigma \rho}+\partial_{\sigma} h_{\nu \rho}-\partial_{\rho} h_{\nu \sigma}\right) .
\end{gathered}
$$

Thus, in the third order we obtain the equation

$$
\frac{\partial P_{++}^{(3)}}{\partial X_{\sigma+}} \partial_{+} \chi_{\sigma}=-2 \chi_{\sigma} h_{\sigma \rho} \partial_{+} X_{\rho+}+2 X_{\sigma+} \delta^{(2)} X_{\sigma+},
$$

where

$$
\delta^{(2)} X_{\sigma+}=-\chi^{\rho}\left(\partial_{+} Y_{\rho \sigma}+\partial_{\rho} X_{\sigma+}+\partial_{\sigma} X_{\rho+}\right)+\frac{\partial_{\sigma}}{\partial_{+}} \chi^{\rho} \partial_{+} X_{\rho+}
$$

enters in the infinitesimal transformation of $X_{\sigma+}$

$$
\delta X_{\sigma+}=\partial_{+} \chi_{\sigma}+\delta^{(2)} X_{\sigma+}
$$

With the integration over $x^{+}$by parts one can rewrite the equation (59) in the form

$$
\frac{\partial P_{++}^{(3)}}{\partial X_{\sigma+}} \partial_{+} \chi_{\sigma}=\left(\partial_{+} \chi_{\sigma}\right) X_{\rho+}\left(Y_{\rho \sigma}+Y_{\sigma \rho}\right)
$$




$$
-2 \chi_{\sigma}\left(\frac{\partial_{\sigma}}{\partial_{+}} X_{\delta+}\right) \partial_{+} X_{\delta+}-2\left(\partial_{\sigma} \chi_{\delta}\right)\left(\frac{1}{\partial_{+}} X_{\sigma+}\right) \partial_{+} X_{\delta+}-2\left(\partial_{+}^{2} \chi_{\delta}\right)\left(\frac{\partial_{\sigma}}{\partial_{+}} X_{\delta+}\right) \frac{1}{\partial_{+}} X_{\sigma+} .
$$

After that its solution can be easily found

$$
P_{++}^{(3)}=X_{\sigma+} X_{\delta+} Y_{\sigma \delta}-2\left(\frac{1}{\partial_{+}} X_{\sigma+}\right)\left(\frac{\partial_{\sigma}}{\partial_{+}} X_{\delta+}\right) \partial_{+} X_{\delta+} .
$$

Integration by parts and using the expression for $Y_{\sigma \delta}$ one can simplify this result

$$
P_{++}^{(3)}=X_{\rho+} X_{\sigma+} h_{\rho \sigma}-X_{\sigma+} \frac{\partial_{\sigma}}{\partial_{+}} X_{\rho+}^{2} .
$$

Therefore we obtain for the effective currents $j_{++}$and $j_{--}$the perturbative expansion

$$
\begin{aligned}
& j_{++}=h_{++}-X_{\sigma+}^{2}+X_{\rho+} X_{\sigma+} h_{\rho \sigma}-X_{\sigma+} \frac{\partial_{\sigma}}{\partial_{+}} X_{\rho+}^{2}+\ldots, \\
& j_{--}=h_{--}-X_{\sigma-}^{2}+X_{\rho-} X_{\sigma-} h_{\rho \sigma}-X_{\sigma-} \frac{\partial_{\sigma}}{\partial_{-}} X_{\rho-}^{2}+\ldots .
\end{aligned}
$$

One can verify, that with our accuracy these currents are transformed under the coordinate change as follows

$$
\delta j_{ \pm \pm} \approx 2 \partial_{ \pm}\left(\chi_{ \pm}-h_{ \pm \sigma} \chi_{\sigma}\right)+\partial_{ \pm}\left(\chi_{\sigma}-h_{\sigma \rho} \chi_{\rho}\right) \frac{\partial_{\sigma}}{\partial_{ \pm}}\left(h_{++}-X_{\sigma+}^{2}\right) .
$$

It allows us to guess the law of transformations of $j_{ \pm \pm}$in a general case

$$
\delta j_{ \pm \pm}=2 \partial_{ \pm} \chi^{\mp}+\partial_{ \pm} \chi^{\sigma} \frac{\partial_{\sigma}}{\partial_{ \pm}} j_{ \pm \pm}, \chi^{\sigma}=g^{\sigma \rho} \chi_{\rho}
$$

\section{Rapidly moving scalar particle in a gravity field}

The general covariance conditions for the effective reggeon currents

$$
\left(\delta h_{\rho \sigma}\right) \frac{\delta}{\delta h_{\rho \sigma}} j_{++}=\left(\delta h_{\rho \sigma}\right) \frac{\delta}{\delta h_{\rho \sigma}} j_{--}=0
$$

are equivalent to equations of motion for the relativistic matter propagating in the corresponding gravitational field because the effective action can be viewed as a backward reaction of the rapidly moving colliding particles on the processes taking place at a given interval of rapidity. Due to the universality of the action for its calculation one can consider an arbitrary type of the colliding matter.

Let us restrict ourselves to the scattering of the high energy scalar particle off the gravitational field. The action for the free massless scalar field $\phi$ in the gravitational background can be written as follows

$$
S_{s}=\int d^{4} x \sqrt{-g} \frac{1}{2}\left(\partial_{\mu} \phi\right) g^{\mu \nu}\left(\partial_{\nu} \phi\right) .
$$


The corresponding energy-momentum tensor is

$$
T_{\mu \nu}=\left(\partial_{\mu} \phi\right)\left(\partial_{\nu} \phi\right)-\frac{1}{2} g_{\mu \nu} g^{\rho \sigma}\left(\partial_{\rho} \phi\right)\left(\partial_{\sigma} \phi\right) .
$$

We introduce the covariant d'Alambert operator

$$
\nabla^{2}=\frac{1}{\sqrt{-g}} \partial_{\mu} g^{\mu \nu} \sqrt{-g} \partial_{\nu}=D^{\mu} \partial_{\mu}
$$

which is symmetric for the following scalar product of the fields

$$
\int d^{4} x \sqrt{-g} \psi \nabla^{2} \phi=\int d^{4} x \sqrt{-g} \phi \nabla^{2} \psi .
$$

The equations of motion for $\phi$ are

$$
\nabla^{2} \phi=0
$$

The energy-momentum tensor is conserved

$$
D^{\mu} T_{\mu \nu}=0
$$

due to the equations of motion.

One can construct also the equation for the Green function $G\left(x, x^{\prime}\right)$ of the scalar particle

$$
-\nabla^{2}(x) G\left(x, x^{\prime}\right)=\delta^{4}\left(x-x^{\prime}\right) .
$$

Its arguments can be interchanged with a similarity transformation

$$
G\left(x, x^{\prime}\right)=\sqrt{-g\left(x^{\prime}\right)} G\left(x^{\prime}, x\right) \frac{1}{\sqrt{-g(x)}} .
$$

The variation of the Green function over the metric tensor can be written as follows

$$
\begin{gathered}
\delta G\left(x, x^{\prime}\right)=\int d^{4} y G(x, y) \delta\left(\sqrt{-g} \nabla^{2}\right) G\left(y, x^{\prime}\right) \\
=-\int d^{4} y \frac{\partial G(x, y)}{\partial y^{\mu}} \delta\left(g^{\mu \nu} \sqrt{-g}\right) \frac{\partial G\left(y, x^{\prime}\right)}{\partial y^{\nu}} .
\end{gathered}
$$

Under the general covariant transformations which can be written in the form

$$
\delta\left(g^{\mu \nu} \sqrt{-g}\right)=\sqrt{-g}\left(g^{\mu \sigma} D_{\sigma} \chi^{\nu}+g^{\mu \sigma} D_{\sigma} \chi^{\nu}-g^{\mu \nu} D_{\sigma} \chi^{\sigma}\right) .
$$

the Green function is transformed as follows

$$
\delta G\left(x, x^{\prime}\right)=\chi^{\sigma}(x) \frac{\partial}{\partial x^{\sigma}} G\left(x, x^{\prime}\right)+\chi^{\sigma}\left(x^{\prime}\right) \frac{\partial}{\partial x^{\prime \sigma}} G\left(x, x^{\prime}\right) .
$$

The corresponding $S$-matrix exists providing that at infinity the metric has the Minkowski form

$$
\lim _{x \rightarrow \infty} g^{\mu \nu}=\eta^{\mu \nu}
$$


In this case the scattering amplitude $f\left(p, p^{\prime}\right)$ is expressed in terms of the matrix element of the Green function with amputated free propagators

$$
f\left(p, p^{\prime}\right) \sim<p^{\prime}\left|\lim _{t \rightarrow \infty} \lim _{t^{\prime} \rightarrow-\infty} \partial_{\sigma}^{2} G\left(x, x^{\prime}\right) \partial_{\sigma^{\prime}}^{2}\right| p>
$$

where the initial and final particles are on mass shell

$$
p^{2}=p^{\prime 2}=0
$$

The scattering amplitude is invariant under the general coordinate transformations, because the infinitesimal parameter $\chi$ tends to zero at infinity. Note, however, that generally the energy and momentum are not conserved.

For our purpose it is enough to find the Green function only at high energies

$$
p_{\sigma} \approx p_{\sigma}^{\prime} \rightarrow \infty
$$

For example let us consider the colliding particle with the momentum

$$
p_{A}=n_{+} \sqrt{\frac{s}{2}} .
$$

In this case the wave functions $<p_{A} \mid$ and $<p_{A^{\prime}} \mid$ are rapidly oscillate and we can write the covariant d'Alambert operator in the equation for $G\left(x, x^{\prime}\right)$ as follows

$$
\nabla^{2}=\partial_{\sigma}^{2}+h^{--} \partial_{-}^{2}+2\left(\partial_{\sigma} h^{\sigma-}\right) \partial_{-}+\frac{1}{2}\left(\partial_{+} h_{\rho \sigma}\right) g^{\rho \sigma} \partial_{-}+\frac{1}{2} h^{\mu-}\left(\partial_{\mu} h_{\rho \sigma}\right) g^{\rho \sigma} \partial_{-},
$$

where we introduced the notations

$$
\begin{gathered}
g^{\rho \sigma}=\eta_{\rho \sigma}+h^{\rho \sigma}, h^{\mu-}=-h_{\mu+}+h_{\mu \rho} h_{\rho+}+\ldots, \\
h^{--}=-h_{++}+h_{+\rho} h_{\rho_{+}}-h_{+\rho} h_{\rho \sigma} h_{\sigma_{+}}+\ldots .
\end{gathered}
$$

We imply also the following decomposition of the usual Laplace operator

$$
\partial_{\sigma}^{2}=\partial_{+} \partial_{-}+\partial_{\sigma} \partial_{\sigma}
$$

The closed expression for the induced current $j_{++}$is given below

$$
j_{++}=\frac{\partial_{\rho}^{2}}{\partial_{-}} \nabla^{-2} \frac{\partial_{\sigma}^{2}}{\partial_{-}},
$$

where it is implied, that the differential operators in the end of the expression act to the left after their integration by parts. One can use also a semiclassical approximation for the Green function. We shall return to the semiclassical approach in another form in subsequent sections. 


\section{5 "Eikonal" contribution to the effective action}

As it was mentioned above, in the perturbation theory the scalar particle in the intermediate states is strongly virtual in an accordance with the fact, that in our kinematics the gravitons emitted from it belong to the clusters with their rapidity significantly different from the particle rapidity. Therefore we can expand its free propagator as follows

$$
-\frac{1}{\partial_{\sigma}^{2}} \approx-\frac{1}{\partial_{+} \partial_{-}}+\frac{1}{\partial_{+} \partial_{-}} \partial_{\sigma}^{\perp} \partial_{\sigma}^{\perp} \frac{1}{\partial_{+} \partial_{-}} .
$$

The leading terms $\sim h^{++}$are canceled partly in the perturbation expansion between contributions of various Feynman diagrams corresponding to a different ordering of the vertices $S_{i n t}$ in time, because the eikonal term with intermediate particles on mass shell in our case is negligible. To clarify this important fact we calculate here several terms of the expansion of scattering amplitude in the Fourier transform $V(k)$ of the interaction term $h_{++}$. Omitting the normalization factors and the vertices $V\left(k_{i}\right)$, the scattering amplitude for the scalar particle with the large momentum $p$ can be written in the second order of perturbation theory as follows

$$
A_{2}^{e i k}=\frac{1}{\left(p+k_{1}\right)^{2}}+\frac{1}{\left(p+k_{2}\right)^{2}}=\frac{\left(p+k_{1}+k_{2}\right)^{2}-2\left(k_{1} k_{2}\right)}{\left(p+k_{1}\right)^{2}\left(p+k_{1}+k_{2}\right)^{2}} \approx-\frac{\left(k_{1} k_{2}\right)}{2\left(p k_{1}\right)\left(p k_{2}\right)},
$$

where we used the reality requirement for the initial and final state particles

$$
p^{2}=\left(p+k_{1}+k_{2}\right)^{2}=0
$$

and the condition of the strong virtuality for the particle in the intermediate states

$$
2\left(p k_{1}\right) \sim 2\left(p k_{2}\right) \gg k_{1}^{2} \sim k_{2}^{2} \sim\left(k_{1} k_{2}\right) .
$$

In an analogous way one can obtain the following contributions from the eikonal diagrams in the third

$$
\begin{aligned}
& A_{3}^{e i k}=\frac{1}{\left(p+k_{1}\right)^{2}} \frac{1}{\left(p+k_{1}+k_{2}\right)^{2}}+\frac{1}{\left(p+k_{2}\right)^{2}} \frac{1}{\left(p+k_{1}+k_{2}\right)^{2}}+\frac{1}{\left(p+k_{1}\right)^{2}} \frac{1}{\left(p+k_{1}+k_{3}\right)^{2}} \\
& \quad+\frac{1}{\left(p+k_{3}\right)^{2}} \frac{1}{\left(p+k_{1}+k_{3}\right)^{2}}+\frac{1}{\left(p+k_{2}\right)^{2}} \frac{1}{\left(p+k_{2}+k_{3}\right)^{2}}+\frac{1}{\left(p+k_{3}\right)^{2}} \frac{1}{\left(p+k_{2}+k_{3}\right)^{2}} \\
& \approx \\
& \frac{k_{3}\left(k_{1}+k_{2}\right)}{4 p k_{3} p\left(k_{1}+k_{2}\right)} \frac{k_{1} k_{2}}{p k_{1} p k_{2}}+\frac{k_{2}\left(k_{1}+k_{3}\right)}{4 p k_{2} p\left(k_{1}+k_{3}\right)} \frac{k_{1} k_{3}}{p k_{1} p k_{3}}+\frac{k_{1}\left(k_{2}+k_{3}\right)}{4 p k_{1} p\left(k_{2}+k_{3}\right)} \frac{k_{2} k_{3}}{p k_{2} p k_{3}}
\end{aligned}
$$

and fourth order

$$
\begin{aligned}
A_{4}^{e i k} & \approx-\frac{k_{1} k_{2}}{8 p k_{1} p k_{2}}\left(\frac{k_{4}\left(k_{1}+k_{2}+k_{3}\right)}{p k_{4} p\left(k_{1}+k_{2}+k_{3}\right)} \frac{k_{3}\left(k_{1}+k_{2}\right)}{p k_{3} p\left(k_{1}+k_{2}\right)}+\frac{k_{3}\left(k_{1}+k_{2}+k_{4}\right)}{p k_{3} p\left(k_{1}+k_{2}+k_{4}\right)} \frac{k_{4}\left(k_{1}+k_{2}\right)}{p k_{4} p\left(k_{1}+k_{2}\right)}\right) \\
& -\frac{k_{1} k_{3}}{8 p k_{1} p k_{3}}\left(\frac{k_{4}\left(k_{1}+k_{2}+k_{3}\right)}{p k_{4} p\left(k_{1}+k_{2}+k_{3}\right)} \frac{k_{2}\left(k_{1}+k_{3}\right)}{p k_{2} p\left(k_{1}+k_{3}\right)}+\frac{k_{2}\left(k_{1}+k_{3}+k_{4}\right)}{p k_{2} p\left(k_{1}+k_{3}+k_{4}\right)} \frac{k_{4}\left(k_{1}+k_{3}\right)}{p k_{4} p\left(k_{1}+k_{3}\right)}\right)
\end{aligned}
$$




$$
\begin{aligned}
& -\frac{k_{1} k_{4}}{8 p k_{1} p k_{4}}\left(\frac{k_{3}\left(k_{1}+k_{2}+k_{4}\right)}{p k_{3} p\left(k_{1}+k_{2}+k_{4}\right)} \frac{k_{2}\left(k_{1}+k_{4}\right)}{p k_{2} p\left(k_{1}+k_{4}\right)}+\frac{k_{2}\left(k_{1}+k_{3}+k_{4}\right)}{p k_{2} p\left(k_{1}+k_{3}+k_{4}\right)} \frac{k_{3}\left(k_{1}+k_{4}\right)}{p k_{3} p\left(k_{1}+k_{4}\right)}\right) \\
& -\frac{k_{2} k_{3}}{8 p k_{2} p k_{3}}\left(\frac{k_{4}\left(k_{1}+k_{2}+k_{3}\right)}{p k_{4} p\left(k_{1}+k_{2}+k_{3}\right)} \frac{k_{1}\left(k_{2}+k_{3}\right)}{p k_{1} p\left(k_{2}+k_{3}\right)}+\frac{k_{1}\left(k_{2}+k_{3}+k_{4}\right)}{p k_{1} p\left(k_{2}+k_{3}+k_{4}\right)} \frac{k_{4}\left(k_{2}+k_{3}\right)}{p k_{4} p\left(k_{2}+k_{3}\right)}\right) \\
& -\frac{k_{2} k_{4}}{8 p k_{2} p k_{4}}\left(\frac{k_{3}\left(k_{1}+k_{2}+k_{4}\right)}{p k_{3} p\left(k_{1}+k_{2}+k_{4}\right)} \frac{k_{1}\left(k_{2}+k_{4}\right)}{p k_{1} p\left(k_{2}+k_{4}\right)}+\frac{k_{1}\left(k_{2}+k_{3}+k_{4}\right)}{p k_{1} p\left(k_{2}+k_{3}+k_{4}\right)} \frac{k_{3}\left(k_{2}+k_{4}\right)}{p k_{3} p\left(k_{2}+k_{4}\right)}\right) \\
& -\frac{k_{3} k_{4}}{8 p k_{3} p k_{4}}\left(\frac{k_{2}\left(k_{1}+k_{3}+k_{4}\right)}{p k_{2} p\left(k_{1}+k_{3}+k_{4}\right)} \frac{k_{1}\left(k_{3}+k_{4}\right)}{p k_{1} p\left(k_{3}+k_{4}\right)}+\frac{k_{1}\left(k_{2}+k_{3}+k_{4}\right)}{p k_{1} p\left(k_{2}+k_{3}+k_{4}\right)} \frac{k_{2}\left(k_{3}+k_{4}\right)}{p k_{2} p\left(k_{3}+k_{4}\right)}\right) \\
& -\frac{\left(k_{3}+k_{4}\right)\left(k_{1}+k_{2}\right)}{8 p\left(k_{3}+k_{4}\right) p\left(k_{1}+k_{2}\right)} \frac{k_{1} k_{2}}{p k_{1} p k_{2}} \frac{k_{3} k_{4}}{p k_{3} p k_{4}}-\frac{\left(k_{2}+k_{4}\right)\left(k_{1}+k_{3}\right)}{8 p\left(k_{2}+k_{4}\right) p\left(k_{1}+k_{3}\right)} \frac{k_{1} k_{3}}{p k_{1} p k_{3}} \frac{k_{2} k_{4}}{p k_{2} p k_{4}} \\
& -\frac{\left(k_{2}+k_{3}\right)\left(k_{1}+k_{4}\right)}{8 p\left(k_{2}+k_{3}\right) p\left(k_{1}+k_{4}\right)} \frac{k_{1} k_{4}}{p k_{1} p k_{4}} \frac{k_{2} k_{3}}{p k_{2} p k_{3}} .
\end{aligned}
$$

The corresponding "eikonal" terms indeed appear in the effective currents

$$
\begin{aligned}
& j_{++}^{e i k} \approx g_{++}-X_{\sigma+}^{2}-X_{\sigma+} \frac{\partial_{\sigma}}{\partial_{+}} X_{\rho+}^{2}-X_{\mu+} \frac{\partial_{\mu}}{\partial_{+}} X_{\sigma+} \frac{\partial_{\sigma}}{\partial_{+}} X_{\rho+}^{2}-\frac{1}{4}\left(\frac{\partial_{\sigma}}{\partial_{+}} X_{\rho+}^{2}\right)^{2}+\ldots, \\
& j_{--}^{e i k} \approx g_{--}-X_{\sigma-}^{2}-X_{\sigma-} \frac{\partial_{\sigma}}{\partial_{-}} X_{\rho-}^{2}-X_{\mu-} \frac{\partial_{\mu}}{\partial_{-}} X_{\sigma-} \frac{\partial_{\sigma}}{\partial_{-}} X_{\rho-}^{2}-\frac{1}{4}\left(\frac{\partial_{\sigma}}{\partial_{-}} X_{\rho-}^{2}\right)^{2}+\ldots,
\end{aligned}
$$

where we took into account, that due to the general covariance the light-cone components $h_{++}$ and $h_{--}$can enter in the final expressions only inside the tensors $X_{\sigma+}$ and $X_{\sigma-}$, respectively.

Looking at these expressions and comparing them with the above perturbative contributions obtained from general covariance considerations one can formulate the hypothesis, that the complete result for the generally invariant currents is obtained from the "eikonal" expression by its "covariantization" corresponding to the substitution of the Minkowski tensor $\eta^{\mu \nu}$ everywhere by the world metric tensor:

$$
\eta^{\mu \nu} \rightarrow g^{\mu \nu} .
$$

This hypothesis leads to the following result in the perturbation theory

$$
\begin{gathered}
j_{++}=h_{++}-X_{\sigma+} g^{\sigma \rho} X_{\rho+}-X_{\sigma+} g^{\sigma \rho} \frac{\partial_{\rho}}{\partial_{+}} X_{\mu+} g^{\mu \nu} X_{\nu+} \\
-X_{\alpha+} g^{\alpha \beta} \frac{\partial_{\beta}}{\partial_{+}} X_{\sigma+} g^{\sigma \rho} \frac{\partial_{\rho}}{\partial_{+}} X_{\mu+} g^{\mu \nu} X_{\nu+}-\frac{g^{\sigma \rho}}{4}\left(\frac{\partial_{\sigma}}{\partial_{+}} X_{\mu+} g^{\mu \nu} X_{\nu+}\right) \frac{\partial_{\rho}}{\partial_{+}} X_{\alpha+} g^{\alpha \beta} X_{\beta+}+\ldots \\
j_{--}=h_{--}-X_{\sigma-} g^{\sigma \rho} X_{\rho-}-X_{\sigma-} g^{\sigma \rho} \frac{\partial_{\rho}}{\partial_{-}} X_{\mu-} g^{\mu \nu} X_{\nu-} \\
-X_{\alpha-} g^{\alpha \beta} \frac{\partial_{\beta}}{\partial_{-}} X_{\sigma-} g^{\sigma \rho} \frac{\partial_{\rho}}{\partial_{-}} X_{\mu-} g^{\mu \nu} X_{\nu-}-\frac{g^{\sigma \rho}}{4}\left(\frac{\partial_{\sigma}}{\partial_{-}} X_{\mu-} g^{\mu \nu} X_{\nu-}\right) \frac{\partial_{\rho}}{\partial_{-}} X_{\alpha-} g^{\alpha \beta} X_{\beta-}+\ldots
\end{gathered}
$$


Moreover, it allows to formulate a closed equation for the important "eikonal" contribution

$$
\begin{gathered}
\left(\partial_{+} \chi_{\rho}\right) \frac{\delta}{\delta X_{\rho+}} j_{++}^{e i k}=\left(\left(\partial_{\rho} \chi_{\sigma}\right)+\left(\partial_{\sigma} \chi_{\rho}\right)\right) \frac{\delta}{\delta \eta_{\rho \sigma}} j_{++}^{e i c} \\
-\left(\chi_{\rho}\left(\partial_{\rho} X_{\sigma+}\right)+\left(\partial_{\sigma} \chi_{\rho}\right) X_{\rho+}-\left(\frac{\partial_{\sigma}}{\partial_{+}}\left(\partial_{+} \chi_{\rho}\right) X_{\rho_{+}}\right)\right) \frac{\delta}{\delta X_{\sigma+}} j_{++}^{e i k},
\end{gathered}
$$

where, as usual, the factors in front of derivatives should substitute in the same position the corresponding variables $X_{\sigma+}$ and $\eta_{\rho \sigma}$ removed by the differentiation. The first term in the right hand side of this equation corresponds to the infinitesimal transformation of $h_{\rho \sigma}$ in the lowest order of the perturbation theory.

For example, in the fourth order from this "eikonal" equation we derive the identity

$$
\begin{gathered}
-\left(\partial_{+} \chi_{\mu}\right) \frac{\partial_{\mu}}{\partial_{+}} X_{\sigma+} \frac{\partial_{\sigma}}{\partial_{+}} X_{\rho+}^{2}-X_{\mu+} \frac{\partial_{\mu}}{\partial_{+}}\left(\partial_{+} \chi_{\sigma}\right) \frac{\partial_{\sigma}}{\partial_{+}} X_{\rho+}^{2} \\
-2 X_{\mu+} \frac{\partial_{\mu}}{\partial_{+}} X_{\sigma+} \frac{\partial_{\sigma}}{\partial_{+}} X_{\rho+} \partial_{+} \chi_{\rho}-\left(\frac{\partial_{\sigma}}{\partial_{+}} X_{\rho+}^{2}\right) \frac{\partial_{\sigma}}{\partial_{+}} X_{\mu+} \partial_{+} \chi_{\mu} \\
\equiv-X_{\sigma+}\left(\left(\partial_{\sigma} \chi_{\rho}\right)+\left(\partial_{\rho} \chi_{\sigma}\right)\right) \frac{\partial_{\rho}}{\partial_{+}} X_{\mu+}^{2}-2 X_{\sigma+} \frac{\partial_{\sigma}}{\partial_{+}} X_{\rho+}\left(\partial_{\rho} \chi_{\delta}\right) X_{\delta+} \\
+\left(\chi_{\rho}\left(\partial_{\rho} X_{\sigma+}\right)+\left(\partial_{\sigma} \chi_{\rho}\right) X_{\rho+}-\left(\frac{\partial_{\sigma}}{\partial_{+}}\left(\partial_{+} \chi_{\rho}\right) X_{\rho+}\right)\right) \frac{\partial_{\sigma}}{\partial_{+}} X_{\mu+}^{2} \\
\left.+2 X_{\mu+} \frac{\partial_{\mu}}{\partial_{+}} X_{\sigma+}\left(\chi_{\rho}\left(\partial_{\rho} X_{\sigma+}\right)+\left(\partial_{\sigma} \chi_{\rho}\right) X_{\rho+}\right)-\frac{\partial_{\sigma}}{\partial_{+}}\left(\partial_{+} \chi_{\rho}\right) X_{\rho+}\right)
\end{gathered}
$$

which can be verified with integration over $x^{+}$by parts.

In the fifth order one can obtain the relation

$$
\begin{gathered}
\left(\partial_{+} \chi_{\rho}\right) \frac{\delta}{\delta X_{\rho+}} P_{++}^{e i k(5)}=-X_{\mu+}\left(\partial_{\mu} \chi_{\nu}\right) \frac{\partial_{\nu}}{\partial_{+}} X_{\sigma+} \frac{\partial_{\sigma}}{\partial_{+}} X_{\rho+}^{2}-X_{\mu+} \frac{\partial_{\mu}}{\partial_{+}} X_{\sigma+}\left(\partial_{\sigma} \chi_{\delta}\right) \frac{\partial_{\delta}}{\partial_{+}} X_{\rho+}^{2} \\
-\frac{1}{2}\left(\partial_{\sigma} \chi_{\rho}\right)\left(\frac{\partial_{\sigma}}{\partial_{+}} X_{\mu+}^{2}\right)\left(\frac{\partial_{\rho}}{\partial_{+}} X_{\nu+}^{2}\right)+\left(\chi_{\rho}\left(\partial_{\rho} X_{\sigma+}\right)-\left(\frac{\partial_{\sigma}}{\partial_{+}}\left(\partial_{+} \chi_{\rho}\right) X_{\rho+}\right)\right) \frac{\partial_{\sigma}}{\partial_{+}} X_{\delta+} \frac{\partial_{\delta}}{\partial_{+}} X_{\mu+}^{2} \\
+X_{\mu+} \frac{\partial_{\mu}}{\partial_{+}}\left(\chi_{\delta}\left(\partial_{\delta} X_{\sigma+}\right)-\left(\frac{\partial_{\sigma}}{\partial_{+}}\left(\partial_{+} \chi_{\delta}\right) X_{\delta+}\right)\right) \frac{\partial_{\sigma}}{\partial_{+}} X_{\rho+}^{2} \\
+\left(2 X_{\mu+} \frac{\partial_{\mu}}{\partial_{+}} X_{\sigma+}+\left(\frac{\partial_{\sigma}}{\partial_{+}} X_{\mu+}^{2}\right)\right) \frac{\partial_{\sigma}}{\partial_{+}} X_{\rho+}\left(\chi_{\delta}\left(\partial_{\delta} X_{\rho+}\right)-\frac{\partial_{\rho}}{\partial_{+}}\left(\partial_{+} \chi_{\delta}\right) X_{\delta+}\right) .
\end{gathered}
$$

It gives a possibility to calculate the corresponding "eikonal" contribution to $j_{++}$in this order

$$
\begin{gathered}
P_{++}^{e i k(5)}=-X_{\nu+} \frac{\partial_{\nu}}{\partial_{+}} X_{\mu+} \frac{\partial_{\mu}}{\partial_{+}} X_{\sigma+} \frac{\partial_{\sigma}}{\partial_{+}} X_{\rho+}^{2} \\
-\frac{1}{4} X_{\nu+} \frac{\partial_{\nu}}{\partial_{+}}\left(\frac{\partial_{\sigma}}{\partial_{+}} X_{\rho+}^{2}\right)^{2}-\frac{1}{2}\left(\frac{\partial_{\sigma}}{\partial_{+}} X_{\mu+} \frac{\partial_{\mu}}{\partial_{+}} X_{\rho+}^{2}\right)\left(\frac{\partial_{\sigma}}{\partial_{+}} X_{\nu+}^{2}\right) .
\end{gathered}
$$


In the sixth order we obtain in a similar way

$$
\begin{gathered}
P_{++}^{e i k(6)}=-X_{\delta+} \frac{\partial_{\delta}}{\partial_{+}} X_{\nu+} \frac{\partial_{\nu}}{\partial_{+}} X_{\mu+} \frac{\partial_{\mu}}{\partial_{+}} X_{\sigma+} \frac{\partial_{\sigma}}{\partial_{+}} X_{\rho+}^{2} \\
-\frac{1}{4} X_{\delta+} \frac{\partial_{\delta}}{\partial_{+}} X_{\nu+} \frac{\partial_{\nu}}{\partial_{+}}\left(\frac{\partial_{\sigma}}{\partial_{+}} X_{\rho+}^{2}\right)^{2}-\frac{1}{2} X_{\delta+} \frac{\partial_{\delta}}{\partial_{+}}\left(\frac{\partial_{\sigma}}{\partial_{+}} X_{\mu+} \frac{\partial_{\mu}}{\partial_{+}} X_{\rho+}^{2}\right)\left(\frac{\partial_{\sigma}}{\partial_{+}} X_{\nu+}^{2}\right) \\
-\frac{1}{4}\left(\frac{\partial_{\sigma}}{\partial_{+}} X_{\mu+} \frac{\partial_{\mu}}{\partial_{+}} X_{\rho+}^{2}\right)^{2}-\frac{1}{8}\left(\frac{\partial_{\sigma}}{\partial_{+}}\left(\frac{\partial_{\delta}}{\partial_{+}} X_{\rho+}^{2}\right)^{2}\right)\left(\frac{\partial_{\sigma}}{\partial_{+}} X_{\nu+}^{2}\right) .
\end{gathered}
$$

To find a general structure for the currents $j_{++}^{e i k}$ and $j_{--}^{e i k}$ we should investigate in a more accurate way the recurrent relation following from the eikonal equation (102).

To begin with, one can use the following formulas (see (60))

$$
\begin{gathered}
2 X_{\sigma_{+}} \delta^{(2)} X_{\sigma+}-X_{\sigma+} X_{\rho_{+}}\left(\partial_{\rho} \chi_{\sigma}+\partial_{\sigma} \chi_{\rho}\right) \\
=-X_{\sigma+} \frac{\partial_{\sigma}}{\partial_{+}} 2 X_{\rho+}\left(\partial_{+} \chi_{\rho}\right)-\left(\partial_{+} \chi_{\sigma}\right) \frac{\partial_{\sigma}}{\partial_{+}} X_{\rho+}^{2}+\partial_{+} \chi_{\sigma} \frac{\partial_{\sigma}}{\partial_{+}} X_{\rho+}^{2}
\end{gathered}
$$

for the variation of the structure $X_{\sigma+}^{2}$ present in the previous order. On the other hand, the sum of the first two terms in the right hand side can be interpreted as the variation of the expression

$$
-X_{\sigma+} \frac{\partial_{\sigma}}{\partial_{+}} X_{\rho+}^{2}
$$

appearing in the next order. The last term in (107) gives a vanishing contribution in the second order. For higher orders it is multiplied with two possible structure $X_{\mu} \partial_{\mu} / \partial_{+}$or $\partial_{\mu} / \partial_{+}$. The second structure is contracted with the index $\mu$ with the operator $\partial_{\mu} / \partial_{+}$acting on another function. Let us consider these two possibilities separately.

We obtain for the variation of the first structure

$$
\begin{gathered}
\delta^{(2)} X_{\mu+} \frac{\partial_{\mu}}{\partial_{+}}-X_{\mu+}\left(\partial_{\nu} \chi_{\mu}+\partial_{\mu} \chi_{\nu}\right) \frac{\partial_{\nu}}{\partial_{+}} \\
=\chi_{\mu} \partial_{\mu} X_{\nu+} \frac{\partial_{\nu}}{\partial_{+}}-X_{\mu+} \partial_{\mu} \chi_{\nu} \frac{\partial_{\nu}}{\partial_{+}}-\left(\frac{\partial_{\nu}}{\partial_{+}}\left(\partial_{+} \chi_{\mu}\right) X_{\mu+}\right) \frac{\partial_{\nu}}{\partial_{+}} .
\end{gathered}
$$

The second term in the right hand side cancels the contribution from the last term in for the variation of $X_{\sigma}^{2}(107)$ due to the relation

$$
-X_{\mu+} \partial_{\mu} \chi_{\nu} \frac{\partial_{\nu}}{\partial_{+}} X_{\sigma+}^{2}+X_{\mu+} \partial_{\mu} \chi_{\sigma} \frac{\partial_{\sigma}}{\partial_{+}} X_{\rho+}^{2}=0
$$

The last term in (109) corresponds to the variation of the following structure in the next order

$$
-\frac{1}{4}\left(\frac{\partial_{\sigma}}{\partial_{+}} X_{\rho+}^{2}\right)^{2}
$$


provided that the operator $X_{\mu+} \frac{\partial_{\mu}}{\partial_{+}}$was applied to $X_{\rho+}^{2}$. In other cases we obtain from the last term the term canceling the variation of the contribution

$$
-\frac{1}{2}\left(\frac{\partial_{\nu}}{\partial_{+}} X_{\mu+}^{2}\right) \frac{\partial_{\nu}}{\partial_{+}}
$$

in the next order. The first term in (109) can be written as follows

$$
-\left(\partial_{+} \chi_{\mu}\right) \frac{\partial_{\mu}}{\partial_{+}} X_{\nu+} \frac{\partial_{\nu}}{\partial_{+}}+\partial_{+} \chi_{\mu} \frac{\partial_{\mu}}{\partial_{+}} X_{\nu+} \frac{\partial_{\nu}}{\partial_{+}}
$$

Here the first contribution leads to the following structure in the next order

$$
-X_{\mu+} \frac{\partial_{\mu}}{\partial_{+}} X_{\nu+} \frac{\partial_{\nu}}{\partial_{+}}
$$

and the second term vanishes provided it is not multiplied by $X_{\mu} \partial_{\mu} / \partial_{+}$or $\partial_{\mu} / \partial_{+}$contracted by the index $\mu$ with the operator $\partial_{\mu} / \partial_{+}$acting on another function. In the these two cases we should repeat calculations performed above for the last term in the variation of $X_{\sigma}^{2}$.

At last we consider the variation of a product of the operators $\partial_{\sigma} / \partial_{+}$in $h_{\sigma \rho}$ of

$$
-\left(\partial_{\sigma} \chi_{\rho}+\partial_{\rho} \chi_{\sigma}\right) \frac{\partial_{\sigma}}{\partial_{+}} \ldots \frac{\partial_{\rho}}{\partial_{+}}=-\left(\partial_{\rho} \chi_{\sigma} \frac{\partial_{\sigma}}{\partial_{+}} \ldots\right) \frac{\partial_{\rho}}{\partial_{+}}-\frac{\partial_{\sigma}}{\partial_{+}} \ldots \partial_{\rho} \chi_{\rho} \frac{\partial_{\rho}}{\partial_{+}}+\chi_{\mu} \partial_{\mu}\left(\frac{\partial_{\sigma}}{\partial_{+}} \ldots \frac{\partial_{\sigma}}{\partial_{+}} \ldots\right) .
$$

Two first terms are canceled with the last terms in Eqs. (107) and (113). The last term can be written as follows

$$
-\left(\partial_{+} \chi_{\mu}\right) \frac{\partial_{\mu}}{\partial_{+}}\left(\frac{\partial_{\sigma}}{\partial_{+}} \ldots \frac{\partial_{\sigma}}{\partial_{+}} \ldots\right)+\partial_{+} \chi_{\mu} \frac{\partial_{\mu}}{\partial_{+}}\left(\frac{\partial_{\sigma}}{\partial_{+}} \ldots \frac{\partial_{\sigma}}{\partial_{+}} \ldots\right)
$$

The first term here corresponds to the following structure in the next order

$$
-X_{\mu+} \frac{\partial_{\mu}}{\partial_{+}}\left(\frac{\partial_{\sigma}}{\partial_{+}} \ldots \frac{\partial_{\sigma}}{\partial_{+}} \ldots\right)
$$

The second term is not zero only if it is multiplied by $X_{\nu} \partial_{\nu} / \partial_{+}$or $\partial_{\nu} / \partial_{+}$contracted by the index $\nu$ with the operator $\partial_{\nu} / \partial_{+}$acting on another function. In these cases again we should perform calculations similar to that with the last term in eq. (107). Thus, after cancelation of some terms in the variation of $j_{++}$in the previous order we can obtain the result in next order, using the above substitutions.

Even more, one can write the following representation for the effective "eikonal" currents in an arbitrary order

$$
j_{++}^{e i k}=h_{++}-\partial_{+} J_{+}^{e i k}, j_{--}=h_{--}-\partial_{-} J_{-}^{e i k} .
$$

The above perturbative calculations allow to construct the following "fan" equations for the quantities $J_{ \pm}^{e i k}$

$$
\left(\partial_{ \pm}-X_{\sigma \pm} \partial_{\sigma}\right) J_{ \pm}^{e i k}=X_{\mu \pm}^{2}+\frac{1}{4}\left(\partial_{\rho} J_{ \pm}^{e i k}\right)^{2}
$$


The solutions of these equations should have the following transformation properties following from the general coordinate invariance of $j_{ \pm \pm}$

$$
\delta J_{ \pm}^{e i k}=-\frac{2}{\partial_{ \pm}} \chi_{\sigma} \partial_{ \pm} X_{\sigma \pm}=\frac{2}{\partial_{ \pm}} X_{\sigma \pm} \partial_{ \pm} \chi_{\sigma}
$$

One can verify, that indeed these properties are compatible with the transformations of various operators entering in the "fan" equations

$$
\begin{gathered}
\delta\left(X_{\sigma \pm} \partial_{\sigma}\right)=\left(\partial_{ \pm} \chi_{\sigma}\right) \partial_{\sigma}-\left(\frac{\partial_{\sigma}}{\partial_{ \pm}}\left(\partial_{ \pm} \chi_{\mu}\right) X_{\mu \pm}\right) \partial_{\sigma}-\left(\partial_{ \pm} \chi_{\mu}\right) \frac{\partial_{\mu}}{\partial_{ \pm}} X_{\sigma \pm} \partial_{\sigma} \\
\delta X_{\mu \pm}^{2}=2 X_{\mu \pm} \partial_{ \pm} \chi_{\mu}-X_{\sigma \pm} \frac{\partial_{\sigma}}{\partial_{ \pm}} 2 X_{\rho \pm}\left(\partial_{ \pm} \chi_{\rho}\right)-\left(\partial_{ \pm} \chi_{\sigma}\right) \frac{\partial_{\sigma}}{\partial_{ \pm}} X_{\rho \pm}^{2}
\end{gathered}
$$

and

$$
\delta \partial_{\rho} \ldots \partial_{\rho} \ldots=-\left(\partial_{ \pm} \chi_{\mu}\right) \frac{\partial_{\mu}}{\partial_{ \pm}}\left(\partial_{\rho} \ldots \partial_{\rho} \ldots\right)
$$

Here we neglected the terms canceled between various structure (see last contributions in Eqs. (107), (113) and (116))

$$
\Delta \delta\left(X_{\sigma \pm} \partial_{\sigma}\right)=-X_{\mu \pm} \partial_{\mu} \chi_{\sigma} \partial_{\sigma}+\partial_{ \pm} \chi_{\mu} \frac{\partial_{\mu}}{\partial_{ \pm}} X_{\sigma \pm} \partial_{\sigma}, \Delta \delta X_{\mu \pm}^{2}=\partial_{ \pm} \chi_{\sigma} \frac{\partial_{\sigma}}{\partial_{ \pm}} X_{\rho \pm}^{2}
$$

and

$$
\Delta \delta \partial_{\rho} \ldots \partial_{\rho} \ldots=-\left(\partial_{\rho} \chi_{\sigma} \partial_{\sigma} \ldots\right) \partial_{\rho}-\partial_{\sigma} \ldots \partial_{\rho} \chi_{\rho} \partial_{\rho}+\partial_{ \pm} \chi_{\mu} \frac{\partial_{\mu}}{\partial_{ \pm}}\left(\partial_{\rho} \ldots \partial_{\rho} \ldots\right)
$$

They generate unessential corrections to $j_{ \pm \pm}$proportional to $\partial_{ \pm}$

$$
\Delta j_{ \pm}=-\partial_{ \pm} \chi_{\sigma} \partial_{\sigma} J_{ \pm}
$$

\section{Hamilton - Jacobi equation for effective currents}

To construct covariant equations for the effective currents in all orders we take into account, that $j_{ \pm \pm}$are invariant under general coordinate transformations up to total derivatives in $x^{ \pm}$. Let us introduce the currents $j^{\mp}$ related directly to $j_{ \pm \pm}$

$$
j^{\mp} \equiv-\frac{1}{\partial_{ \pm}} j_{ \pm \pm}=J_{ \pm}-\frac{1}{\partial_{ \pm}} h_{ \pm \pm}
$$

Using these relations one can transform the "eikonal" equation (119) for $J_{ \pm}^{e i k}$ to the form

$$
-\left(\partial_{ \pm}-h_{\sigma \pm} \partial_{\sigma}\right) j_{e i k}^{\mp}=h_{ \pm \pm}-\left(h_{\rho \pm}\right)^{2}-\frac{1}{4}\left(\partial_{\rho} j_{e i k}^{\mp}\right)^{2} .
$$

In "eikonal" approximation the possible contributions containing the matrix elements $h_{\mu \nu}$ with $\mu, \nu \neq \pm$ are absent. To restore such terms we should impose on the equation the property of general covariance. To begin with, the inhomogeneous term can be modified in 
such a way, that it becomes proportional to a matrix element of the contravariant metric tensor

$$
h_{ \pm \pm}-\left(h_{\rho \pm}\right)^{2} \rightarrow h_{ \pm \pm}-g^{\rho \sigma} h_{\rho \pm} h_{\sigma \pm}=-g^{\mp \mp} .
$$

Here and later the tensors with covariant and contravariant indices are considered to be different. They are related by a contraction with the metric tensor.

Using similar modifications for the linear and quadratic term, one can obtain the generally covariant "fan" equation for the currents $j^{\mp}$

$$
g^{\mp \sigma} \partial_{\sigma} j^{\mp}=g^{\mp \mp}+\frac{g^{\rho \sigma}}{4}\left(\partial_{\sigma} j^{\mp}\right)\left(\partial_{\rho} j^{\mp}\right) .
$$

In an accordance with the general covariance the currents $j^{\mp}$ are transformed as follows

$$
\delta j^{\mp}=2 \chi^{\mp}+\chi^{\rho} \partial_{\rho} j^{\mp},
$$

where the infinitesimal parameters $\chi^{\mp}$ and $\chi^{\rho}$ tend to zero at large $x$ in an accordance with the fact, that $j^{\mp}$ are defined up to the contributions vanishing at $x^{ \pm} \rightarrow \infty$. Indeed, the induced part of the effective action with an integration over $x^{ \pm}$can be written as follows

$$
\Delta S=-\frac{1}{2 \kappa}\left(\int d^{2} x_{\perp} d x^{-} \lim _{x^{+} \rightarrow \infty}\left(j^{-} \frac{\partial_{\mu}^{2} A^{++}}{2}\right)+\int d^{2} x_{\perp} d x^{+} \lim _{x^{-} \rightarrow \infty}\left(j^{+} \frac{\partial_{\mu}^{2} A^{--}}{2}\right)\right)
$$

and this expression is not changed under such transformations.

The equation for $j^{\mp}$ can be presented in a simpler form

$$
g^{\rho \sigma}\left(\frac{1}{2} \partial_{\rho} j^{\mp}-g_{\rho}^{\mp}\right)\left(\frac{1}{2} \partial_{\sigma} j^{\mp}-g_{\sigma}^{\mp}\right)=0 .
$$

Its formal solution is

$$
j^{\mp}=2 x^{\mp}-\omega^{ \pm}
$$

where the quantities $\omega^{ \pm}$satisfy the light front equation

$$
g^{\rho \sigma} \partial_{\rho} \omega^{ \pm} \partial_{\sigma} \omega^{ \pm}=0 .
$$

The last equation can be obtained in an independent way if we would search the solution of the generally covariant d'Alambert equation (see eq. (72) in Section 4)

$$
\nabla^{2} \phi(x)=0
$$

for the wave function of the scalar particle moving with a large momentum $p^{\mp}$ in the semiclassical ansatz

$$
\phi^{ \pm}(x)=\exp \left(-i|p| x^{\mp}+i \theta^{\mp}(x)\right), \theta^{\mp}(x)=\frac{|p|}{2} j^{\mp}(x)
$$

where $\theta^{\mp}(x)$ is a rapidly changing phase and $j^{\mp}$ is the effective current in our normalization. Indeed, by neglecting the derivatives from the metric tensors in comparison with large derivatives from $\phi^{ \pm}$we obtain from the d'Alambert equation its semiclassical version

$$
g^{\rho \sigma}\left(\frac{1}{2} \partial_{\rho} j^{\mp}-g_{\rho}^{\mp}\right)\left(\frac{1}{2} \partial_{\sigma} j^{\mp}-g_{\sigma}^{\mp}\right)=0,
$$


which coincides with the equation (133) for $j^{\mp}$ derived above.

The $S$-matrix for the particle scattering at a given impact parameter off the gravitational field in the semiclassical approximation has the following form

$$
S=\lim _{x^{ \pm} \rightarrow \infty} \exp \left(i \frac{|p|}{2} j^{\mp}(x)\right),
$$

providing that the initial conditions for $j^{\mp}$ are

$$
\lim _{x^{ \pm} \rightarrow-\infty} j^{\mp}(x)=0
$$

In particular this $S$-matrix contains pure eikonal contributions for which the particle in the intermediate states lies on mass shell. Such contributions should be absent in the effective action, although they are reproduced by the iteration of effective vertices in the $s$-channel. It is the reason, why the effective current $j^{\mp}$ entering in the action at large $x^{ \pm}$is proportional to the logarithm of the $S$-matrix

$$
\lim _{x^{ \pm} \rightarrow \infty} j^{\mp}(x)=-i \frac{2}{p^{ \pm}} \ln S .
$$

It is well known [38], that the solution of the light front equation

$$
g^{\rho \sigma} \partial_{\rho} \omega \partial_{\sigma} \omega=0
$$

can be expressed in terms of the null-geodesic trajectories of particles in the gravitational field satisfying the equation of motion

$$
\frac{d^{2} x^{\mu}}{(d \tau)^{2}}=\Gamma_{\alpha \beta}^{\mu} \frac{d x^{\alpha}}{d \tau} \frac{d x^{\beta}}{d \tau},
$$

where $\tau$ is a parameter increasing along the trajectory and $\Gamma_{\alpha \beta}^{\mu}$ is the Christoffel symbol. The geodesic equation is presented in the form of the Hamilton equations

$$
\frac{d x^{\mu}}{d \tau}=g^{\mu \nu} \omega_{\nu}, \frac{d \omega_{\alpha}}{d \tau}=-\frac{1}{2} \omega_{\mu} \omega_{\nu} \partial_{\alpha} g^{\mu \nu}
$$

where

$$
\omega_{\alpha} \equiv \partial_{\alpha} \omega=g_{\alpha \beta} \frac{d x^{\beta}}{d p}
$$

plays a role of the particle momentum.

Note, that the light front equation can be considered as the Hamilton-Jacobi (HJ) equation for the action $\omega$. Its general integral contains an arbitrary function, but it is well known [39], that this general solution is expressed in terms of the so-called complete integral containing only 4 arbitrary constants

$$
\omega=a f\left(x^{\mu}, c_{1}, c_{2}\right)+A
$$

The appearance of the parameters $a$ and $A$ is related to the locality and the homogeneity of the HJ equation (its invariance under the transformation $\omega \rightarrow b \omega+B$ ). Really the HJ 
equation is an integral of motion for the Hamilton equations allowing to find the canonical variables $x^{\mu}$ and $\omega_{\nu}$ as some functions of $\tau$. Indeed, providing that the HJ equation is fulfilled at some $\tau=\tau_{0}$ it will be valid at arbitrary $\tau$ due to the relation

$$
\frac{d}{d \tau} g^{\rho \sigma} \partial_{\rho} \omega \partial_{\sigma} \omega=0
$$

which follows from the Hamilton equations. On the contrary, the general solution of the Hamilton equations can be obtained in terms of the complete integral for $\omega$. Indeed, one can prove [39], that the derivatives of $\omega$ over the parameters $a, c_{1}, c_{2}$

$$
\frac{\partial \omega}{\partial a}=f=d, \frac{\partial \omega}{\partial c_{1}}=d_{1}, \frac{\partial \omega}{\partial c_{2}}=d_{2}
$$

are also integrals of motion and therefore one can find from the last relations the coordinates $x^{i}(i=1,2,3)$ as functions of $t$ and six parameters $a, c_{1}, c_{2}, d, d_{1}, d_{2}$, which corresponds to a general solution of equations of motion.

To construct a complete integral $\omega$ for our case of the massless particle scattering off the gravitation field from the solution of the Hamilton equations we write the light front surface for arbitrary $\tau$ in the form

$$
\omega\left(x^{0}, x^{1}, x^{2}, x^{3}\right)=\text { const } .
$$

Let us assume, that at large distances and large negative times $t_{0}$, where $g^{\mu \nu}=\eta^{\mu \nu}$, this surface is a plane containing the points parametrised by two numbers $u$ and $v$

$$
\vec{x}=t_{0} \vec{n}+u \vec{n}_{1}+v \vec{n}_{2}
$$

where $\vec{n}, \vec{n}_{1}, \vec{n}_{2}$ are orthogonal unit vectors

$$
\vec{n}^{2}=\vec{n}_{1}^{2}=\vec{n}_{2}^{2},\left(\vec{n}, \vec{n}_{1}\right)=\left(\vec{n}, \vec{n}_{2}\right)=\left(\vec{n}_{1}, \vec{n}_{2}\right)=0 .
$$

The initial values of momenta are given below

$$
\vec{\nabla} \omega=c \vec{n}, \omega_{0}^{2}=c^{2},
$$

where $c$ is an arbitrary parameter which depends generally on $u$ and $v$ (note, that $\omega$ is defined up to a common factor). Then from the Hamilton equations one can calculate $x^{\alpha}$ and $\omega_{\beta}$ for all values of $\tau$ and parameters $u$ and $v$. Thus, we can obtain $x^{\alpha}=x^{\alpha}\left(\tau, u, v, t_{0} ; \vec{n}\right)$, which is a parametrised form of the light front surface $\omega^{(n)}=$ const, depending on the light-cone vector

$$
n=\frac{1}{\sqrt{2}}(1, \vec{n}) \text {. }
$$

In the usual form this surface can be obtained by excluding the initial data $\left(u, v, t_{0}\right)$ from four components of the vector $x^{\alpha}$.

In particular, to obtain the effective currents $j^{ \pm}$we should put

$$
\omega^{ \pm}=\omega^{\left(n^{ \pm}\right)}, n^{ \pm}=\frac{1}{\sqrt{2}}(1, \pm 1,0,0)
$$


and normalize the functions $\omega^{ \pm}$in such a way, that

$$
\omega^{ \pm}=2 x^{ \pm}-j^{ \pm}
$$

A possible generalization of the developed effective field theory could include a superposition of the currents $j^{n}$ with different light-cone vectors $n$.

The classical equations for the effective actions apart from the usual Einstein term $G^{\sigma \rho}$ contain the induced terms for the components of the metric tensor $g^{\mp \mp}, g^{\sigma \mp}$ and $g^{\sigma \rho}$. These terms are equal to the corresponding functional derivatives of the action $\Delta S$. The contributions proportional to $A^{++}$and $A^{--}$contain the derivatives from the currents $j^{-}(x)$ and $j^{+}(x)$, respectively. Due to the Hamilton-Jacobi equations these derivatives satisfy the relations

$$
2 g^{\rho \sigma}\left(\partial_{\rho} \omega^{\mp}\right) \partial_{\sigma} \frac{\delta j^{\mp}(x)}{\delta g^{\mu \nu}(y)}=\left(\partial_{\mu} \omega^{\mp}\right)\left(\partial_{\nu} \omega^{\mp}\right) \delta^{4}(x-y) .
$$

The induced terms in the Einstein equation play role of the energy-momentum tensor $T_{\mu \nu}(y)$ which is conserved due to the general covariance of the action $\Delta S$.

\section{Effective action for shock wave gravitational fields}

To illustrate the general approach based on the effective action, let us consider the HamiltonJacobi equation for the massless particle scattering off the gravitation center with the metric tensor given by the Schwarzschild solution [40]

$$
d^{2} s=\left(\frac{r-\alpha}{r+\alpha}\right) d^{2} t-\left(\frac{r+\alpha}{r-\alpha}\right) d^{2} r-(r+\alpha)^{2}\left(d^{2} \theta+\sin ^{2} \theta d^{2} \phi\right),
$$

where we used the spherical coordinates. The parameter $\alpha$ is proportional to the mass $m$ of the attraction center

$$
\alpha=\gamma m, \kappa^{2}=8 \pi \gamma .
$$

The Einstein equations for the massless particle moving around the central body in the plane $(x, y)$ corresponding to $\theta=\pi / 2$ are reduced to two ordinary differential equations [38]

$$
\left(\frac{d r}{d \phi}\right)^{2}=\frac{(r+\alpha)^{4}}{b^{2}}-\left(r^{2}-\alpha^{2}\right),
$$

and

$$
\frac{(r+\alpha)^{3}}{r-\alpha} \frac{d r}{d t}=\sqrt{(r+\alpha)^{4}-\left(r^{2}-\alpha^{2}\right) b^{2}},
$$

where $b$ in our case is the impact parameter of the colliding particle which moves for $t \rightarrow-\infty$ along the line parallel to the axes $x$, which corresponds to the following initial condition for the first equation, describing its trajectory,

$$
r(\phi)_{\left.\right|_{\phi \rightarrow 0}} \approx \frac{b}{\phi} \rightarrow \infty
$$


The solution of this equation can be expressed in terms of the elliptic integral of the first kind

$$
\int_{r}^{\infty} \frac{b d r}{\sqrt{(r+\alpha)^{4}-\left(r^{2}-\alpha^{2}\right) b^{2}}}=\phi .
$$

It allows to find $r$ as a function of $\phi$ and $b$. Inverting this function, one can find $b$

$$
b=b(r, \phi ; \alpha) \text {. }
$$

The solution of the second equation can be written in the form

$$
f(t, r, b, \alpha) \equiv t-\int_{0}^{r} \frac{d r}{\sqrt{(r+\alpha)^{4}-\left(r^{2}-\alpha^{2}\right) b^{2}}} \frac{(r+\alpha)^{3}}{r-\alpha}=C,
$$

where the constant $C$ is found from the initial conditions for $r$ at $t \rightarrow-\infty$. In an accordance with our normalization we can construct the complete integral for the Hamilton-Jacobi equation

$$
\omega^{(n)}=2 f(t, r, b(r, \phi ; \alpha), \alpha),
$$

where the unit vector $\vec{n}$ defines the direction of the initial particle momentum and the impact parameter vector $\vec{\rho}$ is orthogonal to it. The angle $\phi$ is in fact the polar angle with the respect to the vector $\vec{n}$.

To obtain the effective currents $j^{\mp}$ we should put $\vec{n}=\mp \vec{e}_{3}$ and write $\omega$ in the form

$$
\omega^{\mp}=\omega^{\left(n^{\mp}\right)}=2 x^{\mp}-j^{\mp} .
$$

To simplify the perturbative expansion of the effective currents we consider below the massless particle scattering off the gravitation center moving with the relativistic velocity $v \rightarrow c$ in the direction of the third axes $\vec{e}_{3}$. Due to the Lorentz contraction the field of this center is given by the metric corresponding to the shock wave solution of Aichelburg and Sexl

$$
(d s)^{2}=\eta_{\mu \nu} d x^{\mu} d x^{\nu}+h_{--}\left(d x^{-}\right)^{2}
$$

where

$$
h_{--}=\frac{8}{\sqrt{2}} G \mu \ln |\vec{x}| \delta\left(x^{-}\right),
$$

where $\vec{x}$ is the transverse component of the vector $x^{\rho}$.

The Hamilton equation for the particle moving in this field has the form

$$
\frac{d x^{\mu}}{d \tau}=\eta^{\mu \nu} \omega_{\nu}-\delta_{+}^{\mu} h_{--} \omega_{+}, \frac{d \omega_{\alpha}}{d \tau}=\frac{1}{2} \omega_{+} \omega_{+} \partial_{\alpha} h_{--}
$$

Before reaching the shock wave the particle propagates along the straight line

$$
x^{\mu}=x_{0}^{\mu}+\omega_{0}^{\mu} \tau, \omega_{0}^{\mu}=\eta^{\mu \nu}\left(\omega_{\nu}\right)_{0}, \eta_{\mu \nu} \omega_{0}^{\mu} \omega_{0}^{\nu}=0,
$$

where $x_{0}^{\mu}$ and $\left(\omega_{\nu}\right)_{0}$ are initial values of coordinates and momenta. The collision with the moving plane $x^{-}=0$ takes place at the moment $\tau_{c}$ fixed by the equation

$$
z_{c}=t_{c}
$$


where the coordinates of the particle are

$$
z_{c}=z_{0}+\omega_{0}^{3} \tau_{c}, t_{c}=t_{0}+\omega_{0}^{0} \tau_{c}, \vec{\rho}=\vec{x}_{0}-\vec{\omega}_{0} \tau_{c} .
$$

Here we introduced the notation $\vec{\rho}$ for the transverse coordinate $\vec{x}$ at $\tau=\tau_{c}$.

At $\tau>\tau_{c}$ the new values of $\omega_{\alpha}$ are

$$
\omega_{+}=\left(\omega_{+}\right)_{0}, \omega_{-}=\left(\omega_{-}\right)_{0}+\frac{4}{\sqrt{2}} \omega_{+} G \mu \ln \rho \delta\left(x^{-}\right), \vec{\omega}=\vec{\omega}_{0}+\frac{4}{\sqrt{2}} G \mu \frac{\vec{\rho}}{\rho^{2}} \omega_{+},
$$

where $\vec{\rho}$ is fixed by the initial conditions.

From the equation for $x^{\mu}$ we obtain

$$
\begin{gathered}
\omega_{\rho} \omega_{\sigma} \frac{d g^{\rho \sigma}}{d \tau}=\omega_{\rho} \omega_{\sigma}\left(\eta^{\mu \nu} \omega_{\nu}-\delta_{+}^{\mu} h_{--} \omega_{+}\right) \frac{d g^{\rho \sigma}}{d x^{\mu}} \\
=-\omega_{+}^{2}\left(-\frac{8}{\sqrt{2}} G \mu \frac{\vec{\omega} \vec{\rho}}{\rho^{2}} \delta\left(x^{-}\right)+\omega_{+} \frac{8}{\sqrt{2}} G \mu \ln \rho \partial_{-} \delta\left(x^{-}\right)\right) .
\end{gathered}
$$

This relation is compatible with the Hamilton-Jacobi equation

$$
g^{\rho \sigma} \omega_{\rho} \omega_{\sigma}=0
$$

which can be verified by its differentiation in $\tau$ with the use of the Hamilton equation for $\omega_{\alpha}$. From the above explicit expressions for $\omega_{\mu}$ we derive also, that the metric tensor $g^{\rho \sigma}$, calculated in the points of the particle trajectory $x^{\mu}=x^{\mu}(\tau)$ is

$$
g^{\rho \sigma}=\eta^{\rho \sigma}-\delta_{+}^{\rho} \delta_{+}^{\sigma} \frac{8}{\sqrt{2}} G \mu\left(\ln \rho \delta\left(x^{-}\right)-\left(\frac{\vec{\rho} \vec{\omega}_{0}}{\rho^{2} \omega_{+}}+\frac{2}{\sqrt{2}} G \mu \frac{1}{\rho^{2}} \theta\left(x^{-}\right)\right) \theta\left(x^{-}\right)\right) .
$$

The coordinates of the massless particle are

$$
x^{ \pm}=x_{c}^{ \pm}+\omega_{0}^{ \pm}\left(\tau-\tau_{c}\right), \vec{x}=\vec{\rho}-\vec{\omega}_{0}\left(\tau-\tau_{c}\right)
$$

before its collision with the plane wave and

$$
\begin{gathered}
x^{-}=\omega_{+}\left(\tau-\tau_{c}\right), \vec{x}=-\left(\frac{\vec{\omega}_{0}}{\omega_{+}}+\frac{4}{\sqrt{2}} G \mu \frac{\vec{\rho}}{\rho^{2}}\right) \omega_{+}\left(\tau-\tau_{c}\right)+\vec{\rho}, \\
x^{+}=x_{c}^{+}+\left(\frac{\omega_{0}^{+}}{\omega_{+}}+\frac{4}{\sqrt{2}} G \mu\left(\frac{\vec{\omega}_{0} \vec{\rho}}{\omega_{+} \rho^{2}}+\frac{2}{\sqrt{2}} G \mu \frac{1}{\rho^{2}}\right)\right) \omega_{+}\left(\tau-\tau_{c}\right)-\frac{4}{\sqrt{2}} G \mu \ln \rho
\end{gathered}
$$

after its collision at $\tau>\tau_{c}$. Here we used the identity

$$
\int d x^{-} \theta\left(x^{-}\right) \delta\left(x^{-}\right)=\frac{1}{2} .
$$

Note, that the particle moves along the light ray $g_{\rho \sigma} d x^{\rho} d x^{\sigma}=0$.

Finding $\tau-\tau_{c}$ and $\rho$ from two first equations and putting the result in the right hand side of third relation we obtain the complete integral for the corresponding Hamilton-Jacobi equation in our normalization

$$
\omega=2 x^{+}=2 x_{0}^{+}+2 \omega_{0}^{+}\left(\tau-\tau_{c}\right)+j
$$


where the effective current

$$
j=\frac{8}{\sqrt{2}} G \mu\left(\frac{\vec{\omega}_{0} \vec{\rho}}{\omega_{+} \rho^{2}} x^{-}+\frac{2}{\sqrt{2}} G \mu \frac{1}{\rho^{2}} x^{-}-\ln \rho\right) .
$$

Note, that this current can be written as follows

$$
j=-2 \frac{\omega_{0}^{+}}{\omega_{+}} x^{-}+\frac{(\vec{\rho}-\vec{x})^{2}}{x^{-}}-\frac{8}{\sqrt{2}} G \mu \ln \rho
$$

and the equation for $\vec{\rho}$ is simplified

$$
\vec{\partial}_{j}=2 \frac{\vec{\omega}_{0}}{\omega_{+}}
$$

Let us consider the simplest case when the particle colliding with the shock wave has the following initial conditions

$$
\vec{\omega}_{0}=\omega_{0}^{+}=0 .
$$

In this case we have for the effective current

$$
j^{+}=j(g)=\frac{8}{\sqrt{2}} G \mu\left(\frac{2}{\sqrt{2}} G \mu \frac{x^{-}}{\rho^{2}}-\ln \rho\right) .
$$

where the vector $\vec{\rho}$ satisfies the equation

$$
\vec{x}=\vec{\rho}\left(1-\frac{4}{\sqrt{2}} G \mu \frac{x^{-}}{\rho^{2}}\right) .
$$

Its solution is given below

$$
\vec{\rho}=\vec{x} f(z), z=\frac{8}{\sqrt{2}} G \mu \frac{x^{-}}{|x|^{2}},
$$

where

$$
f(z)=\frac{1}{2}(1+\sqrt{1+2 z})=1+\frac{z}{2}-\frac{z^{2}}{4}+\frac{z^{3}}{4}-\frac{5 z^{4}}{16}+\ldots .
$$

The current $j^{+}$can be written in the form

$$
j^{+}=a\left(\frac{1}{4} \frac{|x|^{2}}{|\rho|^{2}} z-\ln \rho\right)=-a(\ln x+\phi(z)), a=\frac{8}{\sqrt{2}} G \mu
$$

where

$$
\phi(z)=\ln f(z)-\frac{1}{4} \frac{z}{f^{2}(z)}=\frac{z}{4}-\frac{z^{2}}{8}+\frac{5}{48} z^{3}-\frac{7}{64} z^{4}+\ldots .
$$

On the other hand, using expressions (97), (98) and (105) for the eikonal currents $j_{ \pm \pm}^{e i k}$ and (48) for $X_{\sigma \pm}$ we can write the current $j^{+}$(127) for the shock wave field (168) in the form

$$
j^{+}=-a \ln x+\frac{a^{2}}{\partial_{-}}\left(\frac{x_{\sigma}}{2 x^{2}}\right)^{2}-\frac{a^{3}}{\partial_{-}} \frac{x_{\mu}}{2 x^{2}} \frac{\partial_{\mu}}{\partial_{-}}\left(\frac{x_{\sigma}}{2 x^{2}}\right)^{2}+\frac{a^{4}}{\partial_{-}} \frac{x_{\nu}}{2 x^{2}} \frac{\partial_{\nu}}{\partial_{-}} \frac{x_{\mu}}{2 x^{2}} \frac{\partial_{\mu}}{\partial_{-}}\left(\frac{x_{\sigma}}{2 x^{2}}\right)^{2}
$$




$$
\begin{gathered}
+\frac{a^{4}}{4 \partial_{-}}\left(\frac{\partial_{\mu}}{\partial_{-}}\left(\frac{x_{\sigma}}{2 x^{2}}\right)^{2}\right)^{2}-\frac{a^{5}}{\partial_{-}} \frac{x_{\rho}}{2 x^{2}} \frac{\partial_{\rho}}{\partial_{-}} \frac{x_{\nu}}{2 x^{2}} \frac{\partial_{\nu}}{\partial_{-}} \frac{x_{\mu}}{2 x^{2}} \frac{\partial_{\mu}}{\partial_{-}}\left(\frac{x_{\sigma}}{2 x^{2}}\right)^{2} \\
-\frac{a^{5}}{4 \partial_{-}} \frac{x_{\nu}}{2 x^{2}} \frac{\partial_{\nu}}{\partial_{-}}\left(\frac{\partial_{\mu}}{\partial_{-}}\left(\frac{x_{\sigma}}{2 x^{2}}\right)^{2}\right)^{2}-\frac{a^{5}}{2 \partial_{-}}\left(\frac{\partial_{\mu}}{\partial_{-}} \frac{x_{\nu}}{2 x^{2}} \frac{\partial_{\nu}}{\partial_{-}}\left(\frac{x_{\sigma}}{2 x^{2}}\right)^{2}\right)\left(\frac{\partial_{\mu}}{\partial_{-}}\left(\frac{x_{\sigma}}{2 x^{2}}\right)^{2}\right) .
\end{gathered}
$$

Differentiating over $x_{\sigma}$ and integrating over $x^{+}$we obtain the same expression for $j^{-}$, which can serve as a verification of the approach.

Let us consider now a more general situation of the massless scattering off the gravitational field with the metric

$$
g_{\mu \nu}=\eta_{\mu \nu}+\delta_{\mu}^{-} \delta_{\nu}^{-} V(\vec{x}) \delta\left(x^{-}\right),
$$

where the potential $V$ is an arbitrary function of the points on the shock plane. Repeating the above calculation, we obtain the generalized equation for the point $\vec{\rho}$ in which the particle crosses the plane

$$
\vec{x}=\vec{\rho}-\frac{x^{-}}{2} \vec{\partial} V(\vec{\rho})
$$

and the expression for the effective current $j^{+}$

$$
j^{+}=-V(\vec{\rho})+\frac{x^{-}}{4}(\vec{\partial} V(\vec{\rho}))^{2}=-V(\vec{\rho})+\frac{(\vec{\rho}-\vec{x})^{2}}{x^{-}} .
$$

Note, that the equation for the point $\vec{\rho}$ can be written as the stationarity condition for $j^{+}$ as a function of $\vec{\rho}$

$$
\vec{\partial} j^{+}=0
$$

Using the perturbation theory for the solution of the equation for $\epsilon_{\mu}=\rho_{\mu}-x_{\mu}$ in metric $\eta_{\mu \nu}$

$$
\epsilon_{\mu}=\frac{x^{-}}{2} V_{\mu}-\frac{x^{-}}{2} V_{\mu \mu_{1}} \frac{x^{-}}{2} V_{\mu_{1}}+2 \frac{x^{-}}{2} V_{\mu \mu_{1}} \frac{x^{-}}{2} V_{\mu_{1} \mu_{2}} \frac{x^{-}}{2} V_{\mu_{2}}+\ldots
$$

where

$$
V_{\mu_{1}, \mu_{2}, \ldots \mu_{n}} \equiv \partial_{\mu_{1}} \partial_{\mu_{2} \ldots,} \partial_{\mu_{n}} V(\vec{x})
$$

and putting the result in $j^{+}$, we find

$$
j_{e i k}^{+}=-V(x)+x^{-}\left(\frac{1}{2} V_{\sigma}\right)^{2}+\frac{\left(x^{-}\right)^{2}}{2} V_{\mu} \partial_{\mu}\left(\frac{1}{2} V_{\sigma}\right)^{2}+\ldots
$$

in an agreement with the expressions (98) for the eikonal contribution with the simplified expression for $X_{\sigma-}$

$$
X_{\sigma-} \rightarrow-\frac{\partial_{\rho}}{\partial_{-}} g_{--}
$$




\section{Variational principle for the effective currents}

Let us consider even more general configuration of the gravitational field consisting from $n$ shock waves moving in the $z$-direction

$$
g_{\mu \nu}=\eta_{\mu \nu}+\delta_{\mu}^{-} \delta_{\nu}^{-} \sum_{r=1}^{n} V^{(r)}(\vec{x}) \delta\left(x^{-}-x_{r}^{-}\right)
$$

where $x_{r}^{-}$are some parameters ordered in the following way

$$
x_{1}^{-}<x_{2}^{-}<\ldots<x_{n}^{-} .
$$

By solving the Hamilton equations for the massless particle flying at $\tau \rightarrow-\infty$ along the $z$ axes from $z=-\infty$ with the impact parameter $\vec{\rho}$ and $\omega_{0}^{+}=\vec{\omega}_{0}=0$ for each of the intervals $x_{r}^{-}<x^{-}<x_{r+1}^{-}$for $r=1,2, \ldots, n$ we obtain for the points $\vec{\rho}_{r}$ in which the trajectory crosses the corresponding planes the following recurrence relation

$$
\begin{aligned}
& \vec{\rho}_{1}=\vec{\rho}, \vec{\rho}_{2}=\vec{\rho}_{1}-\frac{x_{2}^{-}-x_{1}^{-}}{2} \vec{\partial}_{1} V^{(1)}\left(\vec{\rho}_{1}\right), \vec{\rho}_{3}=\vec{\rho}_{2}-\frac{x_{3}^{-}-x_{2}^{-}}{2} \sum_{t=1}^{2} \vec{\partial}_{t} V^{(t)}\left(\vec{\rho}_{t}\right), \ldots \\
& \vec{\rho}_{n}=\vec{\rho}_{n-1}-\frac{x_{n}^{-}-x_{n-1}^{-}}{2} \sum_{t=1}^{n-1} \vec{\partial}_{t} V^{(t)}\left(\vec{\rho}_{t}\right), \vec{x}=\vec{\rho}_{n}-\frac{x^{-}-x_{n}^{-}}{2} \sum_{t=1}^{n} \vec{\partial}_{t} V^{(t)}\left(\vec{\rho}_{t}\right), \quad
\end{aligned}
$$

where $\vec{x}$ and $x^{-}$are coordinates of the particle after its interaction with all shock waves. Note, that the $x^{-}$-coordinate of the particle and its momentum $\omega_{+}$are not changed during collisions

$$
x^{-}=\omega_{+} \tau+x_{0}^{-} .
$$

But the momenta $\omega_{+}$and $\vec{\omega}$ are different in each interval $x_{r}^{-}<x^{-}<x_{r+1}^{-}$

$$
\omega_{-}=\frac{\omega_{+}}{2} \sum_{r=1}^{n} V^{(r)}\left(\vec{\rho}_{r}\right) \delta\left(x^{-}-x_{r}^{-}\right), \vec{\omega}=\vec{\omega}_{r}=\frac{\omega_{+}}{2} \sum_{t=1}^{r} \vec{\partial}_{t} V^{(t)}\left(\vec{\rho}_{t}\right) .
$$

The metric tensor, calculated on the particle trajectory in this interval, has the form

$$
g^{\rho \sigma}=\eta^{\rho \sigma}-\delta_{+}^{\rho} \delta_{+}^{\sigma} \sum_{r=1}^{n}\left(V^{(r)}\left(\vec{\rho}_{r}\right) \delta\left(x^{-}-x_{r}^{-}\right)-\frac{\theta^{2}\left(x^{-}-x_{r}^{-}\right)}{4} \sum_{t=1}^{r}\left(\vec{\partial}_{t} V^{(t)}\left(\vec{\rho}_{t}\right)\right)^{2}\right),
$$

compatible with the integral of motion

$$
g^{\rho \sigma} \omega_{\rho} \omega_{\sigma}=2 \omega_{+} \omega_{-}-\vec{\omega}^{2}+\omega_{+}^{2} g^{++}=0 .
$$

Note, that the total derivative of $g^{++}$in $\tau$ is in an agreement with the Hamilton equations

$$
\frac{d g^{++}}{d \tau}=-\omega_{+} \partial_{-} g^{++}+\frac{2 \vec{\omega}}{\omega_{+}^{2}} \frac{d \vec{w}}{d \tau}
$$

The coordinate $x^{+}$is also changed after each collision and after all collisions we have

$$
x^{+}=x_{0}^{+}+\frac{1}{2} \sum_{r=1}^{n}\left(x_{r+1}^{-}-x_{r}^{-}\right)\left(\sum_{t=1}^{r} \frac{\overrightarrow{\partial t}_{t}}{2} V^{(t)}\left(\vec{\rho}_{t}\right)\right)^{2}-\frac{1}{2} \sum_{r=1}^{n} V^{(r)}\left(\vec{\rho}_{r}\right),
$$


where it is implied, that $\vec{\rho}_{n+1}=\vec{x}$ and $x_{n+1}^{-}=x^{-}$. Thus, we obtain for the corresponding effective current in the above gravitational field the following expression

$$
j^{+}=\sum_{r=1}^{n}\left(x_{r+1}^{-}-x_{r}^{-}\right)\left(\sum_{t=1}^{r} \frac{\vec{\partial}_{t}}{2} V^{(t)}\left(\vec{\rho}_{t}\right)\right)^{2}-\sum_{r=1}^{n} V^{(r)}\left(\vec{\rho}_{r}\right),
$$

where it is assumed, that the points $\vec{\rho}_{r}$ are expressed in terms of $\vec{x}$ and $x_{-}$with the use of equations (202). Due to these equations the effective current can be written even in a simpler form

$$
j^{+}=\sum_{r=1}^{n} \frac{\left(\vec{\rho}_{r+1}-\vec{\rho}_{r}\right)^{2}}{x_{r+1}^{-}-x_{r}^{-}}-\sum_{r=1}^{n} V^{(r)}\left(\vec{\rho}_{r}\right), \vec{\rho}_{n+1}=\vec{x}, x_{n+1}^{-}=x^{-} .
$$

Such form of the effective current gives a possibility to write the equations for $\vec{\rho}_{r}$ as its stationarity conditions

$$
\vec{\partial}_{r} j^{+}=0 \text {. }
$$

One can verify the perturbative expansion of this effective current by comparing it with the general expressions (98) for the eikonal contribution.

Let us consider the continuous limit of the scattering problem, assuming that the number of shock waves is infinite and the distance between them tends to zero. In this case the metric tensor on the particle trajectory is

$$
g^{\rho \sigma}\left(\vec{x}, x^{-}\right)=\eta^{\rho \sigma}-\delta_{+}^{\rho} \delta_{+}^{\sigma}\left(g^{++}\left(\vec{\rho}, x^{-}\right)+\frac{\vec{\omega}^{2}}{\omega_{+}^{2}}\right),
$$

where

$$
\vec{\omega}=\omega_{+} \partial_{-} \vec{\rho}
$$

and $\vec{\rho}$ is considered to be a function of $x_{-}$and $\vec{x}$ calculated with the use of the equation of motion for the colliding particle. The effective current can be written in the integral form

$$
j^{+}=\int_{-\infty}^{x^{-}} d y^{-}\left(g^{++}\left(y^{-}, \vec{\rho}\left(y^{-}\right)\right)+\left(\partial_{-} \vec{\rho}\right)^{2}\right)
$$

where the variable $y^{-}$enumerates the shock waves.

This functional can be considered as a classical action for the particle moving in the gravitational field, which allows to formulate the variational principle for the effective current $j^{+}$. Indeed, $j^{+}$should be calculated on the particle geodesic trajectory $\vec{\rho}\left(x^{-}, \vec{x}\right)$. The trajectory are found from the stationarity conditions for this functional which have the form of the non-relativistic Newton equations

$$
2 \partial_{-}^{2} \vec{\rho}=\vec{\partial} g^{++}
$$

Note, that the "potential" $g^{++}$depends explicitly on $x_{-}$and therefore the energy, which is a formal integral of motion for this equation, is not conserved. But with taking into account, that the partial derivatives in $x^{-}$of $\omega_{-}$and $g^{++}$are proportional, we can write the correct integral of motion in the form

$$
\left(\partial_{-} \vec{\rho}\right)^{2}-g^{++}-2 \frac{\omega_{-}}{\omega_{+}}=0
$$


which is really coincides with the Hamilton-Jacobi equation. Indeed, the variation over $\vec{\rho}$ in the integrand for $j^{-}$after the use of the stationarity equations gives a total derivative over $x^{-}$leading after its integration to the relation

$$
\delta j^{+}=2\left(\partial_{-} \vec{\rho}\right) \delta \vec{\rho}
$$

and therefore we have the relation

$$
\partial_{-} \vec{\rho}=\frac{1}{2} \vec{\partial} j^{+}=-\frac{\vec{w}}{\omega_{+}} .
$$

As a result, the integral of motion (216) coincides with the HY equation for this case.

Therefore we obtain the non-linear equation for $j^{+}$compatible with the above variational principle

$$
j^{+}=\int_{-\infty}^{x^{-}} d y^{-}\left(g^{++}\left(y^{-}, \vec{\rho}\left(y^{-}\right)\right)+\frac{1}{4}\left(\vec{\partial} j^{+}\right)^{2}\right) .
$$

Here the functions $\vec{\rho}\left(y^{-}\right)$) are solutions of the Hamilton equations. The iteration of this equation over $g^{++}$reproduces results (98) for the pure eikonal contribution

$$
j_{\text {eik }}^{+}=\frac{1}{\partial_{-}} g^{++}-\frac{1}{\partial_{-}}\left(\frac{1}{2} \frac{\vec{\partial}}{\partial_{-}} g^{++}\right)^{2}+\frac{1}{\partial_{-}}\left(\frac{1}{2} \frac{\vec{\partial}}{\partial_{-}} g^{++}\right) \frac{\vec{\partial}}{\partial_{-}}\left(\frac{1}{2} \frac{\vec{\partial}}{\partial_{-}} g^{++}\right)^{2}+\ldots
$$

where the contributions from the expansion of $g^{++}$in $\vec{\rho}$ with the subsequent use of the hamilton equation and the integration by parts are also taken into account.

As we argued in the previous sections, the effective currents $j^{ \pm}$as functionals of the metric tensors in a general form satisfy the Hamilton-Jacobi equation (see (130))

$$
g^{\sigma \pm} \partial_{\sigma} j^{ \pm}=g^{ \pm \pm}+\frac{g^{\sigma \rho}}{4}\left(\partial_{\sigma} j^{ \pm}\right)\left(\partial_{\rho} j^{ \pm}\right)
$$

It would be important to write the solution of this equation as an extremum of an local functional similar to (214), because in the perturbative expansion $(65,66)$ this locality property is lost. Moreover, such functional could help us in finding quantum-mechanical corrections to the effective action and its supersymmetric generalization. For this purpose one should present (214) in the form invariant under the general covariant transformations. We hope to return to this problem in our future publications.

\section{$9 \quad$ Effective reggeon-graviton vertices}

Let us apply the effective action to the problem of calculations of the simplest effective vertices for the reggeon-graviton interactions in the lowest order of the perturbation theory. For

this purpose it is enough to leave in the currents $j_{ \pm \pm}$only two first terms of the perturbative expansion

$$
j_{ \pm \pm} \approx h_{ \pm \pm}-X_{\sigma \pm}^{2}, X_{\sigma \pm}=h_{\sigma \pm}-\frac{1}{2} \frac{\partial_{\sigma}}{\partial_{ \pm}} h_{ \pm \pm}
$$


We expand also the Christoffel symbol

$$
\Gamma_{\mu \nu}^{\rho} \approx \frac{1}{2}\left(\partial_{\mu} h_{\rho \nu}+\partial_{\nu} h_{\rho \mu}-\partial_{\rho} h_{\mu \nu}-h_{\rho \sigma}\left(\partial_{\mu} h_{\sigma \nu}+\partial_{\nu} h_{\sigma \mu}-\partial_{\sigma} h_{\mu \nu}\right)\right)
$$

and the Hilbert-Einstein Lagrangian

$$
\sqrt{-g} R=\sqrt{-g} g^{\mu \nu}\left(\partial_{\nu} \Gamma_{\mu \rho}^{\rho}-\partial_{\rho} \Gamma_{\mu \nu}^{\rho}+\Gamma_{\mu \rho}^{\sigma} \Gamma_{\sigma \nu}^{\rho}-\Gamma_{\mu \nu}^{\sigma} \Gamma_{\sigma \rho}^{\rho}\right) \approx L_{2}+L_{3},
$$

where

$$
L_{2}=\frac{\partial_{\sigma} h_{\mu \sigma}}{2}\left(\partial_{\mu} h_{\rho \rho}-\partial_{\rho} h_{\mu \rho}\right)+\frac{1}{4}\left(\left(\partial_{\sigma} h_{\mu \nu}\right)^{2}-\left(\partial_{\sigma} h_{\mu \mu}\right)^{2}\right)
$$

and

$$
\begin{gathered}
L_{3}=h_{\rho \sigma}\left(\left(\partial_{\mu} h_{\mu \sigma}\right) \partial_{\nu} h_{\nu \rho}-\frac{\partial_{\rho} h_{\mu \nu}}{4} \partial_{\sigma} h_{\mu \nu}-\frac{\partial_{\mu} h_{\nu \sigma}}{2}\left(\partial_{\nu} h_{\mu \rho}+\partial_{\mu} h_{\rho \nu}\right)+\frac{\partial_{\mu} h_{\mu \nu}}{2}\left(2 \partial_{\rho} h_{\nu \sigma}-\partial_{\nu} h_{\rho \sigma}\right)\right) \\
+h_{\rho \rho}\left(h_{\mu \nu}\left(\partial_{\mu} \partial_{\sigma} h_{\nu \sigma}-\frac{1}{2} \partial_{\sigma}^{2} h_{\mu \nu}\right)+\frac{1}{2}\left(\partial_{\nu} h_{\nu \sigma}\right)^{2}-\frac{3}{8}\left(\partial_{\sigma} h_{\mu \nu}\right)^{2}+\frac{1}{4}\left(\partial_{\sigma} h_{\mu \nu}\right) \partial_{\mu} h_{\sigma \nu}\right) \\
-\frac{h_{\rho \rho}}{8}\left(\partial_{\nu} h_{\sigma \sigma}\right)^{2}-\frac{h_{\rho \rho}}{4} h_{\mu \nu} \partial_{\mu} \partial_{\nu} h_{\sigma \sigma} .
\end{gathered}
$$

These expressions are valid up to the terms proportional to total derivatives which give vanishing contributions to the action $S_{H E}$.

The action is invariant under the general coordinate transformations

$$
\delta S_{H E}=0
$$

with the same accuracy, which can be verified by checking the following relations

$$
\delta L_{2}=\partial_{\nu}\left(\frac{h_{\mu \mu}}{2}\left(\partial_{\sigma}^{2} \chi_{\nu}-\partial_{\nu} \partial_{\sigma} \chi_{\sigma}\right)+h_{\rho \mu} \partial_{\rho} \partial_{\nu} \chi_{\mu}-h_{\nu \mu} \partial_{\rho}^{2} \chi_{\mu}\right)+\chi_{\mu} a_{\mu}, \delta L_{3}=-\chi_{\mu} a_{\mu},
$$

where

$$
\begin{gathered}
a_{\mu} \approx \frac{\partial_{\sigma}^{2} h_{\nu \nu}}{2} \partial_{\mu} h_{\rho \rho}-\left(\partial_{\sigma}^{2} h_{\nu \nu}\right) \partial_{\delta} h_{\delta \mu}-\frac{\partial_{\mu} h_{\nu \nu}}{2} \partial_{\sigma} \partial_{\rho} h_{\rho \sigma}+\frac{\partial_{\delta} \partial_{\nu} h_{\rho \rho}}{2}\left(\partial_{\nu} h_{\delta \mu}+\partial_{\delta} h_{\nu \mu}-\partial_{\mu} h_{\delta \nu}\right) \\
+\left(\partial_{\sigma} \partial_{\nu} h_{\sigma \nu}\right) \partial_{\rho} h_{\rho \mu}+\left(\frac{\partial_{\sigma}^{2} h_{\rho \nu}}{2}-\partial_{\rho} \partial_{\delta} h_{\nu \delta}\right)\left(\partial_{\nu} h_{\rho \mu}+\partial_{\rho} h_{\nu \mu}-\partial_{\mu} h_{\rho \nu}\right)
\end{gathered}
$$

Because the induced contributions to the action are also generally covariant, the EulerLagrange equations for the total action are self-consistent. We can write them in the form

$$
R^{\mu \nu}-\frac{1}{2} g^{\mu \nu} R=\frac{1}{2} \frac{\delta}{\delta g_{\mu \nu}} \int d^{4} x\left(j_{++} \partial_{\sigma}^{2} A^{++}+j_{--} \partial_{\sigma}^{2} A^{--}\right)
$$

where in the right hand side it is implied as usual, that the calculation of the variational derivative over $g_{\mu \nu}$ is combined with the corresponding integration by parts. The solution of these equations can be expanded in the series over the reggeon fields $A_{\text {干干 }}$

$$
\bar{h}_{\mp \mp}=A_{\mp \mp}+O\left(A^{2}\right)
$$


similar to the case of the Euler-Lagrange equation for the effective action in QCD [23]. Inserting this solution in the effective action one can obtain various effective vertices for the self-interaction of the reggeon fields $A_{\mp \mp}$ in the tree approximation. The physical gravitational fields will correspond to the fluctuations $\delta h=h-\bar{h}$ around the classical solution. The functional integration over these fluctuations in the quadratic approximation will lead to the graviton Regge trajectories and to various reggeon couplings in the one-loop approximation. This traditional approach will be considered in future publications. Here we restrict ourselves to the simple cases where the results can be obtained in the lowest orders of perturbation theory.

To begin with, we note, that performing the functional gaussian integration over $h_{\mu \nu}$ from the exponent containing the induced action with the terms linear $h_{++}$and $h_{--}$we obtain the kinetic term for the fields $A_{ \pm \pm}$

$$
-\frac{1}{2 \kappa} \int \frac{d^{4} x}{2}\left(-\partial_{\sigma} h_{++} \partial_{\sigma} h_{--}-h_{++} \partial_{\sigma}^{2} A_{--}-h_{--} \partial_{\sigma}^{2} A_{++}\right) \rightarrow-\frac{1}{2 \kappa} \int d^{4} x \frac{\partial_{\sigma} A_{++} \partial_{\sigma} A_{--}}{2} .
$$

The kinetic term for the reggeon fields should have an opposite sign. Therefore we include in the effective action the bare kinetic term for the reggeon fields

$$
S_{k i n}=\frac{1}{2 \kappa} \int d^{4} x \partial_{\sigma} A_{++} \partial_{\sigma} A_{--}
$$

to have the correct renormalized contribution. Strictly speaking the propagator of the reggeized graviton should contain the $\theta$-function corresponding to the ordering of rapidities $y$ in the different clusters

$$
<0 \mid\left(A_{--}^{y_{1}}\left(x_{1}\right) A_{++}^{y_{2}}\left(x_{2}\right)\right)=4 \frac{\kappa}{\pi^{2}} \theta\left(y_{1}-y_{2}\right) \frac{i}{\left(x_{1}-x_{2}\right)^{2}} .
$$

Further, the next order corrections in each of the induced actions

$$
-\frac{1}{2 \kappa} \int \frac{d^{4} x}{2}\left(-\left(h_{++}-\frac{1}{4}\left(\frac{\partial_{\rho}}{\partial_{+}} h_{++}\right)^{2}\right) \partial_{\sigma}^{2} A_{--}-\left(h_{--}-\frac{1}{4}\left(\frac{\partial_{\rho}}{\partial_{-}} h_{--}\right)^{2}\right) \partial_{\sigma}^{2} A_{++}\right)
$$

lead with the use of the gaussian integration over the fields $h_{ \pm \pm}$to the cubic interactions of the reggeon fields

$$
S^{1 \rightarrow 2}=-\frac{1}{2 \kappa} \int \frac{d^{4} x}{8}\left(\left(\frac{\partial_{\rho}}{\partial_{+}} A_{++}\right)^{2} \partial_{\sigma}^{2} A_{--}+\left(\frac{\partial_{\rho}}{\partial_{-}} A_{--}\right)^{2} \partial_{\sigma}^{2} A_{++}\right) .
$$

Note, that the usual triple graviton vertex gives a vanishing contribution to this interaction.

In an analogous way one can calculate in the tree approximation the effective action for the reggeon transitions $1 \rightarrow n$

$$
S^{1 \rightarrow n}=-\frac{1}{2 \kappa} \int \frac{d^{4} x}{2}\left(\partial_{+} J_{+}^{e i k}\left(A_{++}\right) \partial_{\sigma}^{2} A_{--}+\partial_{-} J_{-}^{e i k}\left(A_{--}\right) \partial_{\sigma}^{2} A_{++}\right),
$$

where the "eikonal" currents $J_{ \pm}^{e i k}\left(h_{ \pm \pm}\right)$can be obtained from the solution of the "fan" equations

$$
\left(\partial_{ \pm}+\frac{1}{2}\left(\frac{\partial_{\sigma}}{\partial_{ \pm}} h_{ \pm \pm}\right) \partial_{\sigma}\right) J_{ \pm}^{e i k}=\frac{1}{4}\left(\frac{\partial_{\sigma}}{\partial_{ \pm}} h_{ \pm \pm}\right)^{2}+\frac{1}{4}\left(\partial_{\rho} J_{ \pm}^{e i k}\right)^{2}
$$


The effective action for the reggeon transitions $2 \rightarrow n(n \geq 2)$ in the same approximation contains a contribution from the usual triple graviton vertex. The general reggeon interaction $n \rightarrow m$ is expressed in terms of the solution of the Euler-Lagrange equation for the effective theory.

Let us consider now the effective action for the reggeon-reggeon-graviton (RRG) interaction in a tree approximation $S^{R R G}$. It contains the contribution from the triple graviton vertex (gv) and from the second order (so) correction $\left(\sim h^{2}\right)$ to the induced action

$$
S^{R R G}=\frac{1}{2 \kappa} \int d^{4} x L^{R R G}, L^{R R G}=L_{g v}^{R R G}+L_{s o}^{R R G},
$$

where

$$
\begin{gathered}
L_{g v}^{R R G}=A_{++}\left(\left(\partial_{\mu} h_{\mu+}-\frac{\partial_{-} h_{++}}{2}\right) \partial_{-} A_{--}-\left(\partial_{+} h_{\mu-}+\partial_{\mu} h_{-+}+\frac{\partial_{\nu} h_{\nu \mu}}{2}\right) \partial_{\mu} A_{--}\right) \\
+A_{--}\left(\left(\partial_{\mu} h_{\mu-}-\frac{\partial_{+} h_{--}}{2}\right) \partial_{+} A_{++}-\left(\partial_{-} h_{\mu+}+\partial_{\mu} h_{-+}+\frac{\partial_{\nu} h_{\nu \mu}}{2}\right) \partial_{\mu} A_{++}\right) \\
\quad-h_{\rho \sigma} \frac{\partial_{\rho} A_{++}}{2} \partial_{\sigma} A_{--}-h_{+-}\left(\partial_{-} A_{--}\right) \partial_{+} A_{++}-h_{+-}\left(\partial_{\nu} A_{++}\right) \partial_{\nu} A_{--} \\
+h_{\rho \rho}\left(-\frac{1}{2} A_{++} \partial_{\sigma}^{2} A_{--}-\frac{1}{2} A_{--} \partial_{\sigma}^{2} A_{++}-\frac{3}{4}\left(\partial_{\sigma} A_{++}\right) \partial_{\sigma} A_{--}+\frac{1}{2}\left(\partial_{+} A_{++}\right) \partial_{-} A_{--}\right) .
\end{gathered}
$$

and

$$
\begin{aligned}
& L_{s o}^{R R G}=\left(-\left(h_{-+}-\frac{1}{2} \frac{\partial_{-}}{\partial_{+}} h_{++}\right) A_{++}+\frac{1}{2}\left(h_{\sigma+}-\frac{1}{2} \frac{\partial_{\sigma}}{\partial_{+}} h_{++}\right)\left(\frac{\partial_{\sigma}}{\partial_{+}} A_{++}\right)\right) \partial_{\rho}^{2} A_{--} \\
& +\left(-\left(h_{-+}-\frac{1}{2} \frac{\partial_{+}}{\partial_{-}} h_{--}\right) A_{--}+\frac{1}{2}\left(h_{\sigma-}-\frac{1}{2} \frac{\partial_{\sigma}}{\partial_{-}} h_{--}\right)\left(\frac{\partial_{\sigma}}{\partial_{-}} A_{--}\right)\right) \partial_{\rho}^{2} A_{++}
\end{aligned}
$$

The effective action $S^{R R G}$ is invariant

$$
\delta S^{R R G}=0
$$

under the "abelian" part of the general covariant transformation

$$
\delta h_{\rho \sigma}=\partial_{\rho} \chi_{\sigma}+\partial_{\sigma} \chi_{\rho}
$$

because the corresponding contributions $S_{g v}^{R R G}$ and $S_{s o}^{R R G}$ are transformed as follows (cf. (228) and (229))

$$
\begin{gathered}
\delta S_{g v}^{R R G}=-\delta S_{s o}^{R R G}=\frac{1}{2 \kappa} \int d^{4} x \Phi(x) \\
\Phi(x)=\left(-\chi_{-} \partial_{+} A_{++}+\frac{1}{2} \chi_{\sigma} \partial_{\sigma} A_{++}\right) \partial_{\rho}^{2} A_{--}+\left(-\chi_{+} \partial_{-} A_{--}+\frac{1}{2} \chi_{\sigma} \partial_{\sigma} A_{--}\right) \partial_{\rho}^{2} A_{++} .
\end{gathered}
$$

For the field of the produced graviton on the mass shell we have additional constraints

$$
\partial_{\mu}^{2} h_{\rho \sigma}=\partial_{\mu} h_{\mu \rho}=h_{\rho \rho}=0
$$


and the RRG lagrangian is simplified as follows

$$
\begin{gathered}
L^{R R G}=A_{++}\left(-\frac{\partial_{-} h_{++}}{2} \partial_{-} A_{--}-\left(\partial_{+} h_{\mu-}+\partial_{\mu} h_{-+}\right) \partial_{\mu} A_{--}\right) \\
+A_{--}\left(-\frac{\partial_{+} h_{--}}{2} \partial_{+} A_{++}-\left(\partial_{-} h_{\mu+}+\partial_{\mu} h_{-+}\right) \partial_{\mu} A_{++}\right) \\
+\left(-\left(h_{-+}-\frac{1}{2} \frac{\partial_{-}}{\partial_{+}} h_{++}\right) A_{++}+\frac{1}{2}\left(h_{\sigma+}-\frac{1}{2} \frac{\partial_{\sigma}}{\partial_{+}} h_{++}\right)\left(\frac{\partial_{\sigma}}{\partial_{+}} A_{++}\right)\right) \partial_{\rho}^{2} A_{--} \\
+\left(-\left(h_{-+}-\frac{1}{2} \frac{\partial_{+}}{\partial_{-}} h_{--}\right) A_{--}+\frac{1}{2}\left(h_{\sigma-}-\frac{1}{2} \frac{\partial_{\sigma}}{\partial_{-}} h_{--}\right)\left(\frac{\partial_{\sigma}}{\partial_{-}} A_{--}\right)\right) \partial_{\rho}^{2} A_{++}
\end{gathered}
$$

Moreover, the corresponding RRG vertex can be written in the momentum space as follows $[32,33]$

$$
\Gamma_{\mu \nu}^{R R G}\left(q_{2}, q_{1}\right)=\frac{1}{2} C_{\mu}\left(q_{2}, q_{1}\right) C_{\nu}\left(q_{2}, q_{1}\right)-\frac{1}{2} N_{\mu}\left(q_{2}, q_{1}\right) N_{\nu}\left(q_{2}, q_{1}\right) .
$$

Here $C\left(q_{2}, q_{1}\right)$ is the effective vertex describing the gluon production from the reggeized gluon

$$
C\left(q_{2}, q_{1}\right)=-q_{1}^{\perp}-q_{2}^{\perp}+p_{A}\left(\frac{q_{1}^{2}}{k p_{A}}+\frac{k p_{B}}{p_{A} p_{B}}\right)-p_{B}\left(\frac{q_{2}^{2}}{k p_{B}}+\frac{k p_{A}}{p_{A} p_{B}}\right),
$$

where $q_{1}, q_{2}$ are the momenta of the reggeized gluons, $k=q_{1}-q_{2}$ is the momentum of the produced gluon and $p_{A}, p_{B}$ are the momenta of the colliding particles. The vector $N\left(q_{2}, q_{3}\right)$ is proportional to the photon bremstrahlung factor in QED

$$
N\left(q_{2}, q_{1}\right)=\sqrt{q_{1}^{2} q_{2}^{2}}\left(\frac{p_{A}}{p_{A} k}-\frac{p_{B}}{p_{B} k}\right) .
$$

Using the light-cone gauge for the polarization tensor of the produced graviton the RRG vertex can be written in a simple form, which allows one to construct the corresponding term in the effective action for the scattering amplitude with the multi-regge unitarity [35].

Let us consider now the effective action for the graviton scattering off the reggeized gravitons. It can be written as a sum of two terms

$$
S^{G G R}=\frac{1}{2 \kappa} \int d^{4} x\left(L^{G G R}\left(A_{++}\right)+L^{G G R}\left(A_{--}\right)\right)
$$

proportional to $A_{++}$and $A_{--}$, respectively. We consider only the first term, because the second one can be obtained from it by interchanging the light-cone indices + and - . In turn, $L^{G G R}\left(A_{++}\right)$is the sum of contributions from the triple reggeon vertex (rv) and the second order (so) correction to the induced term

$$
L^{G G R}\left(A_{++}\right)=L_{g v}^{A_{++}}+L_{s o}^{A_{--}},
$$


where

$$
\begin{aligned}
L_{g v}^{A_{++}}= & A_{++}\left(\left(\partial_{\mu} h_{\mu-}\right)^{2}-\frac{\left(\partial_{-} h_{\mu \nu}\right)^{2}}{4}-\frac{\partial_{\mu} h_{\nu-}}{2}\left(\partial_{\nu} h_{\mu-}+\partial_{\mu} h_{\nu-}\right)+\frac{\partial_{\mu} h_{\mu \nu}}{2}\left(2 \partial_{-} h_{\nu-}-\partial_{\nu} h_{--}\right)\right) \\
& -\left(\partial_{\rho} A_{++}\right)\left(\frac{h_{\rho \sigma}}{2} \partial_{\sigma} h_{--}+h_{\sigma-}\left(\partial_{-} h_{\rho \sigma}+\partial_{\rho} h_{\sigma-}\right)-h_{\rho-} \partial_{\sigma} h_{\sigma-}+\frac{h_{--}}{2} \partial_{\sigma} h_{\sigma \rho}\right) \\
+h_{\rho \rho}( & \left(-A_{++}\left(\frac{\partial_{\sigma}^{2} h_{--}}{2}+\frac{\partial_{-}^{2} h_{\sigma \sigma}}{4}\right)-\frac{h_{--}}{2} \partial_{\sigma}^{2} A_{++}+\left(\partial_{\sigma} A_{++}\right)\left(\frac{\partial_{-} h_{\sigma-}}{2}-3 \frac{\partial_{\sigma} h_{--}}{4}\right)\right) \quad(251)
\end{aligned}
$$

and

$$
L_{s o}^{A_{++}}=-\frac{1}{2}\left(h_{\rho-}-\frac{1}{2} \frac{\partial_{\rho}}{\partial_{-}} h_{--}\right)^{2} \partial_{\sigma}^{2} A_{++} .
$$

The corresponding lagrangians are transformed under the general coordinate transformations as follows

$$
\begin{gathered}
\delta L_{g v}^{A_{++}}=-\chi_{\rho}\left(\left(\partial_{-} h_{\rho-}-\frac{1}{2} \partial_{\rho} h_{--}\right) \partial_{\sigma}^{2} A_{++}-\left(\partial_{-}^{2} h_{\sigma \sigma}+\partial_{\sigma}^{2} h_{--}-2 \partial_{-} \partial_{\sigma} h_{\sigma-}\right) \frac{\partial_{\rho} A_{++}}{2}\right) \\
-\chi_{-}\left(\partial_{-} \partial_{\rho} h_{\sigma \sigma}+\partial_{\sigma}^{2}-\partial_{-} \partial_{\sigma} h_{\rho \sigma}-\partial_{\rho} \partial_{\sigma} h_{\sigma-}\right) \partial_{\rho} A_{++}
\end{gathered}
$$

and

$$
\delta L_{s o}^{A_{++}}=\chi_{\rho}\left(\partial_{-} h_{\rho-}-\frac{1}{2} \partial_{\rho} h_{--}\right) \partial_{\sigma}^{2} A_{++}
$$

We can simplify the GGR lagrangian providing that gravitons are on the mass shell and their fields satisfy additional constraints (244)

$$
\begin{gathered}
L^{G G R}\left(A_{++}\right)=A_{++}\left(-\frac{\left(\partial_{-} h_{\mu \nu}\right)^{2}}{4}-\frac{\partial_{\mu} h_{\nu-}}{2}\left(\partial_{\nu} h_{\mu-}+\partial_{\mu} h_{\nu-}\right)\right) \\
-\left(\partial_{\rho} A_{++}\right)\left(\frac{h_{\rho \sigma}}{2} \partial_{\sigma} h_{--}+h_{\sigma-}\left(\partial_{-} h_{\rho \sigma}+\partial_{\rho} h_{\sigma-}\right)\right)-\frac{1}{2}\left(h_{\rho-}-\frac{1}{2} \frac{\partial_{\rho}}{\partial_{-}} h_{--}\right)^{2} \partial_{\sigma}^{2} A_{++} .
\end{gathered}
$$

The corresponding vertex for the graviton scattering off the reggeon field $A_{++}$can be written as follows (see ref. [32, 33])

$$
\Gamma_{\mu \nu, \mu^{\prime} \nu^{\prime}}^{G G R}=\frac{1}{2}\left(\Gamma_{\mu \mu^{\prime}}^{G G R} \Gamma_{\nu \nu^{\prime}}^{G G R}+\Gamma_{\mu \nu^{\prime}}^{G G R} \Gamma_{\nu \mu^{\prime}}^{G G R}\right),
$$

where $\Gamma_{\mu \mu^{\prime}}^{G G R}$ is the effective vertex for the gluon scattering off the reggeized gluon field $A_{+}$

$$
\Gamma_{\mu \mu^{\prime}}^{G G R}=-\left(\eta_{\mu \mu^{\prime}}-\frac{k_{\mu}^{\prime} p_{\mu^{\prime}}^{B}+k_{\mu^{\prime}} p_{\mu}^{B}}{k p^{B}}-q^{2} \frac{p_{\mu}^{B} p_{\mu^{\prime}}^{B}}{2\left(k p^{B}\right)^{2}}\right),
$$

where $k$ and $k^{\prime}$ are momenta of the initial and final gluons, $p^{B}$ is the momentum of the another initial gluon and $q$ is the momentum transfer. After the transition to the helicity basis the above vertex $\Gamma_{\mu \nu, \mu^{\prime} \nu^{\prime}}^{G G R}$ corresponds to the conservation of the graviton helicity and leads to the corresponding contribution in the effective action for the scattering amplitude with the multi-Regge unitarity [35]. 


\section{Graviton Regge trajectory and supergravity}

To calculate the graviton Regge trajectory in one loop [32] it is needed to contract two GGR vertices appearing in $L^{G G R}\left(A_{++}\right)$and $L^{G G R}\left(A_{--}\right)$with two graviton propagators and integrate the product over the loop momentum. The integration over the Sudakov variables $\alpha$ and $\beta$ of the virtual graviton momentum should give $\ln s$ equal to the relative rapidity of the initial particles. To obtain a non-trivial $s$-dependence in each of two GGR lagrangians one should leave only the singular contributions appearing in the induced terms

$$
L^{G G R}\left(A_{ \pm \pm}\right) \approx\left(\frac{1}{2} h_{\rho \mp} \frac{\partial_{\rho}}{\partial_{\mp}} h_{\mp \mp}-\frac{1}{8}\left(\frac{\partial_{\rho}}{\partial_{\mp}} h_{\mp \mp}\right)^{2}\right) \partial_{\sigma}^{2} A_{ \pm \pm} .
$$

From these expressions one can derive the scattering amplitude described by the contribution of the box diagrams corresponding to two graviton exchange in the crossing channel

$$
F=\delta_{\lambda_{A} \lambda_{A^{\prime}}} \delta_{\lambda_{B} \lambda_{B^{\prime}}} \frac{\kappa_{L}^{4} s^{2}}{(2 \pi)^{4} i} \int \frac{d^{2} k_{\perp} d k_{+} d k_{-}}{\left(k_{\perp}^{2}+2 k_{+} k_{-}+i \epsilon\right)\left((q-k)_{\perp}^{2}+2 k_{+} k_{-}+i \epsilon\right)} f(k, q),
$$

where $\lambda_{r}$ are the helicities of the scattered particles and the function $f(k, q)$ is given below

$$
\begin{gathered}
f(k, q)=\frac{1}{2} \frac{(k, q-k)^{2}}{\left(k_{+} k_{-}\right)^{2}}+\frac{k^{2}+(q-k)^{2}+4(k, q-k)}{k_{+} k_{-}} \\
=\frac{\left(k_{\perp}, q_{\perp}-k_{\perp}\right)^{2}}{4\left(k_{+}-i \epsilon\right)^{2}}\left(\frac{1}{\left(k_{-}+i \epsilon\right)^{2}}+\frac{1}{\left(k_{-}-i \epsilon\right)^{2}}\right)+\frac{q_{\perp}^{2}}{2\left(k_{+}-i \epsilon\right)}\left(\frac{1}{k_{-}+i \epsilon}+\frac{1}{k_{-}-i \epsilon}\right) .
\end{gathered}
$$

Here we restored the analytic structure of the poles in an accordance with the Feynman $i \epsilon$-prescription. The integral over $k_{+}$in $F$ is non-zero only for $k_{-}>0$. Taking it by residues with the subsequent integration over $k_{-}$one can obtain

$$
F=F_{\text {Born }} \omega(t) \ln s, t=q_{\perp}^{2},
$$

where

$$
F_{\text {Born }}=\delta_{\lambda_{A} \lambda_{A^{\prime}}} \delta_{\lambda_{B} \lambda_{B^{\prime}}} \kappa^{2} \frac{s^{2}}{t}
$$

is the scattering amplitude in the Born approximation and $j=2+\omega(t)$ is the graviton Regge trajectory [32]

$$
\omega\left(q_{\perp}^{2}\right)=\frac{\kappa^{2}}{(2 \pi)^{3}} \int \frac{q_{\perp}^{2} d^{2} k_{\perp}}{k_{\perp}^{2}(q-k)_{\perp}^{2}}\left(\frac{(k, q-k)_{\perp}^{2}}{k_{\perp}^{2}}+\frac{(k, q-k)_{\perp}^{2}}{(q-k)_{\perp}^{2}}-q_{\perp}^{2}-\frac{N}{2}(k, q-k)_{\perp}\right) .
$$

Here we added the contribution of $N$ gravitinos for the $N$-extended supergravity [32]. Other super-partners do not give any contribution in this order.

Note, that the infrared divergency of the Regge trajectory is universal, but the logarithmic divergency at large $k_{\perp}$ depends on $N$ and is absent at $N=4$. Really the sum of the one-loop diagrams do not contain any ultraviolet divergency, because the gravity is renormalized in one loop. It means, that the integral over $k_{\perp}^{2}$ is restricted from above by the value of the order of $s$, which leads to the double-logarithmic asymptotics of the scattering amplitude 
with the graviton quantum numbers in the $t$-channel. In Ref. [32] the corresponding ladder diagrams in the double-logarithmic approximation were summed and the following result for the amplitude in the $N$-extended supergravity was obtained

$$
A_{2 \rightarrow 2}=-\kappa^{2} \frac{s^{2}}{t} \delta_{\lambda_{A} \lambda_{A^{\prime}}} \delta_{\lambda_{B} \lambda_{B^{\prime}}} \frac{1}{a \xi} I_{1}(2 a \xi),
$$

where $\lambda_{i}$ are helicities of the initial and final gravitons, $I_{n}(x)$ is the modified Bessel function and the parameters $a$ and $\xi$ are given below

$$
a=\left((4-N) \frac{\kappa^{2}}{16 \pi^{2}}(-t)\right)^{\frac{1}{2}}, \xi=\frac{s}{t} .
$$

In principle there could be double-logarithmic contributions from other diagrams containing three and more gravitons in the $t$-channel. To investigate this possibility one should generalize the effective action constructed above to the supersymmetric case, because the contribution of the superpartners of the graviton is essential for its Regge trajectory in higher loops. But we consider below for simplicity only the first non-trivial correction to the action in the $N=1$ supergravity. In this case apart from the vierbein $e_{\mu}^{m}$, related to the metric tensor $g_{\mu \nu}$ in the well-known way

$$
g_{\mu \nu}=\sum_{n} e_{\mu n} e_{\nu}^{n}
$$

the Rarita-Schwinger field $\psi_{\mu}$ describing the gravitino with the spin $3 / 2$ is introduced. The action for this field is given below

$$
S_{3 / 2}=\int d^{4} x L_{3 / 2}, \quad L_{3 / 2}=-\frac{1}{2} \epsilon^{\mu \nu \rho \sigma} \bar{\psi}_{\mu} \gamma_{5} \gamma_{\nu} D_{\rho} \psi_{\sigma}
$$

The covariant derivative $D_{\rho}$ is defined by the relation

$$
D_{\rho}=\partial_{\rho}+\frac{1}{2} \sigma_{m n} \omega_{\rho}^{m n}, \sigma_{m n}=\frac{1}{2}\left(\gamma_{m} \gamma_{n}-\gamma_{n} \gamma_{m}\right)
$$

where $\omega_{\rho}^{m n}$ is the spin connection expressed in terms of the Christoffel symbol

$$
\begin{gathered}
\omega_{\rho}^{m n}=-e^{\sigma n} \partial_{\rho} e_{\sigma}^{m}+e^{\sigma n} e_{\alpha}^{m} \Gamma_{\rho \sigma}^{\alpha} \\
=\frac{1}{2} e^{\sigma n}\left(\partial_{\sigma} e_{\rho}^{m}-\partial_{\rho} e_{\sigma}^{m}\right)-\frac{1}{2} e^{\sigma m}\left(\partial_{\sigma} e_{\rho}^{n}-\partial_{\rho} e_{\sigma}^{n}\right)+\frac{1}{2} e^{\nu n} e^{\mu m} e^{\rho k}\left(\partial_{\nu} e_{\mu}^{k}-\partial_{\mu} e_{\nu}^{k}\right) .
\end{gathered}
$$

The total action of supergravity is invariant under the supersymmetry transformation

$$
\delta e_{\mu}^{m}=\frac{\kappa}{2} \bar{\epsilon} \gamma^{m} \psi_{\mu}, \delta \psi_{\mu}=\frac{1}{\kappa} D_{\mu} \epsilon
$$

where $\epsilon$ is a local parameter of these transformations being the anticommuting Majorano spinor. It is known, that to close the SUSY commutator algebra off-shell one should introduce the auxiliary fields $S, P$ and $A_{m}$. Here for simplicity of discussion we do not take into account them neglecting total derivatives in the action and in its variation. 
Let us start again with the Born contribution to the induced contribution to the effective lagrangian

$$
L_{i n d}=-\frac{1}{4 \kappa^{2}}\left(j_{++} \partial_{\sigma}^{2} A_{--}+j_{--} \partial_{\sigma}^{2} A_{++}\right), j_{ \pm \pm} \approx h_{ \pm \pm}+\ldots
$$

and attempt to add to it radiative corrections in the fields $h_{\mu \nu}$ and $\psi_{\mu}$ to derive its generalization invariant under the local supersymmetric transformations.

We obtain the following infinitesimal transformation of the metric tensor with the light cone components

$$
\delta h_{ \pm \pm}=\kappa \bar{\epsilon} \gamma_{ \pm} \psi_{ \pm}
$$

To cancel this term one should add to $j_{ \pm \pm}$the contribution

$$
\Delta_{1} j_{ \pm \pm}=\frac{\kappa^{2}}{2} \bar{\psi}_{ \pm} \frac{\gamma_{ \pm}}{\partial_{ \pm}} \psi_{ \pm}
$$

because up to a total derivative in the integrand for the action its supersymmetric transformation is equal to the expression

$$
\delta_{1}\left(\Delta_{1} j_{ \pm \pm}\right) \approx-\kappa \bar{\epsilon} \gamma_{ \pm} \psi_{ \pm}
$$

opposite to $\delta h_{ \pm \pm}$in sign.

Thus, in the $N=1$ supersymmetric gravity we obtain for $j_{ \pm \pm}$with the next-to-leading accuracy The following result

$$
j_{ \pm \pm} \approx h_{ \pm \pm}-X_{\sigma \pm}^{2}+\frac{\kappa^{2}}{2} \bar{\psi}_{ \pm} \frac{\gamma_{ \pm}}{\partial_{ \pm}} \psi_{ \pm}+\ldots
$$

The upper order corrections can be calculated in a similar way.

\section{Discussion}

In this paper the effective action for the high energy processes in gravity was constructed in terms of the currents $j^{ \pm}$satisfying the Hamilton-Jacobi equation. This equation can be solved in the perturbation theory or for simple configurations of the external gravitational fields. One can formulate a variational principle for the currents calculated at such fields. The effective action can be used for the calculation of various elastic and inelastic scattering amplitudes in the Regge kinematics. The Feynman rules for the simple vertices containing the reggeized gravitons are extracted from the effective lagranjian. The one loop graviton Regge trajectory does not contain the ultraviolet divergency only in the $\mathrm{N}=4$ supergravity. In other models the amplitudes with the graviton quantum numbers in the crossing channel have the double-logarithmic terms. It is possible, that the constructed effective action can be generalized to the case of superstrings living in the anti-de-Sitter 10-dimensional space. In this case one could use it for the discription of the Pomeron interactions at the $\mathrm{N}=4$ supersymmetric gauge theory in the framework of the AdS/CFT correspondence. 


\section{Acknowledgements}

I thank the Hamburg University for the hospitality and J. Bartels, E. Levin, A. Sabio Vera and A. Prygarin for helpful discussions. This work was supported by the grant RFFI-10-0201338-a.

\section{References}

[1] V. N. Gribov, Sov. Phys. JETP 14478 (1962).

[2] S. Mandelstam, Nuovo Cim. 30, 1148 (1963).

[3] V. N. Gribov, I. Ya. Pomeranchuk and K. A. Ter-Martirosyan, Phys. Rev. B 139, 184 (1965).

[4] V. N. Gribov, Sov. Phys. JETP 26, 414 (1968).

[5] M. Gell-Mann, M. L. Goldberger, F. E. Low, E. Marx and F. Zachariasen, Phys. Rev. 133, B145 (1954).

[6] S. Mandelstam, Phys. Rev. 137, B949 (1965).

[7] M. T. Grisaru, H. J. Schnitzer and H.S. Tsao, Phys. Rev. Lett. 30, 811, (1973).

[8] L. N. Lipatov, Sov. J. Nucl. Phys. 23338 (1976);

V. S. Fadin, E. A. Kuraev, L. N. Lipatov, Phys. Lett. B 6050 (1975);

E. A. Kuraev, L. N. Lipatov, V. S. Fadin, Sov. Phys. JETP 44443 (1976).

[9] L. N. Lipatov, Phys. Lett. B 309394 (1993).

[10] L. N. Lipatov, Sov. Phys. JETP 63904 (1986).

[11] Bartels, J., Nucl. Phys. B175 (1980) 365;

Kwiecinskii, J., Praszalowicz, M., Phys. Lett. B94 (1980) 413.

[12] L. N. Lipatov High energy asymptotics of multi-colour QCD and exactly solvable lattice models, hep-th/9311037, unpublished.

[13] L. N. Lipatov, Nucl. Phys. B 548328 (1999).

[14] V. S. Fadin, L. N. Lipatov, Phys. Lett. B 429127 (1998);

M. Ciafaloni and G. Camici, Phys. Lett. B 430349 (1998).

[15] A. V. Kotikov, L. N. Lipatov, Nucl. Phys. B 58219 (2000).

[16] A. V. Kotikov, L. N. Lipatov, Nucl. Phys. B 66119 (2003).

[17] A. V. Kotikov, L. N. Lipatov, A. I. Onishchenko, V. N. Velizhanin, Phys. Lett. B 595 521 (2004); [Erratum-ibid. B 632754 (2006)]. 
[18] L. N. Lipatov, talk at "Perspectives in Hadronic Physics", Proc. of Conf. ICTP, Triest, Italy, May 1997.

[19] J. M. Maldacena, Adv. Theor. Math. Phys. 2231 (1998).

[20] S. S. Gubser, I. R. Klebanov, A. M. Polyakov, Phys. Lett. B 428105 (1998).

[21] E. Witten, Adv. Theor. Math. Phys. 2253 (1998).

[22] R. C. Brower, J. Polchinsky, M. J. Strassler, C. I. Tan, JHEP 0712005 (2007).

[23] L. N. Lipatov, Nucl. Phys. B B 452, 369 (1995); Phys. Rept. 286, 131 (1997).

[24] E. N. Antonov, L. N. Lipatov, E. A. Kuraev, I. O. Cherednikov, Nucl. Phys. B 721, $111(2005)$.

[25] Z. Bern, L. J. Dixon, V. A. Smirnov, Phys. Rev. D 72, 085001 (2005).

[26] J. Bartels, L. N. Lipatov, A. Sabio Vera, Phys. Rev. D 80, 045002 (2009).

[27] J. Bartels, L. N. Lipatov, A. Sabio Vera, Eur. Phys. J. C 65 587, (2009),

[28] L. N. Lipatov, preprint, hep-th 1008.1015.

[29] L. N. Lipatov, A. Prygarin, preprints, hep-th 1008.1016, hep-th 1011.2673.

[30] L. N. Lipatov, J. Phys. A 42, 304020 (2009).

[31] M. T. Grisaru, B. van Nieuwenhuizen and C. C. Wu, Phys. Rev D 12 1563; M. T. Grisaru, and H. J. Schnitzer, Phys. Lett 107B 196 (1981).

[32] L. N. Lipatov, Phys. Lett. 116B, 411 (1982).

[33] L. N. Lipatov, JETP, 82, 991 (1982).

[34] A. Bellini, M. Ademollo, M. Ciafaloni, Nucl.Phys. B393, 79 (1993).

[35] L. N. Lipatov, Nucl. Phys. B365, 614 (1991).

[36] D. Amati, M. Ciafaloni and G. Veneziano, JHEP 0802, 049 (2008).

[37] S. B. Giddings, M. Schmidt-Sommerfeld and J. R. Andersen, preprint, hep-th 1005.5408.

[38] V. Fock, The theory of space, time and gravitation, Pergamon Press, London and Aylesbury (1969).

[39] L. D. Landau, E. M. Lifshitz, Mechanics, Course of Theoretical Physics, Butterworth Heinemann.

[40] K. Schwarzschild, S. B. Preuss. Acad. Wiss. 189 (1916). 